\title{
The star formation law at GMC scales in M33, the Triangulum galaxy
}

\author{
Thomas G. Williams, ${ }^{\star}$ Walter K. Gear and Matthew W. L. Smith \\ School of Physics \& Astronomy, Cardiff University, Queens Buildings, The Parade, Cardiff, CF24 3AA, UK
}

Accepted 2018 May 25. Received 2018 May 25; in original form 2018 February 13

\begin{abstract}
We present a high spatial resolution study, on scales of $\sim 100 \mathrm{pc}$, of the relationship between star formation rate (SFR) and gas content within Local Group galaxy M33. Combining deep SCUBA-2 observations with archival GALEX, SDSS, WISE, Spitzer, and submillimetre Herschel data, we are able to model the entire SED from UV to sub-mm wavelengths. We calculate the SFR on a pixel-by-pixel basis using the total infrared luminosity, and find a total SFR of $0.17 \pm 0.06 \mathrm{M}_{\odot} \mathrm{yr}^{-1}$, somewhat lower than our other two measures of SFR - combined FUV and $24 \mu \mathrm{m}$ SFR $\left(0.25_{-0.07}^{+0.10} \mathrm{M}_{\odot} \mathrm{yr}^{-1}\right)$ and SED-fitting tool MAGPHYs $\left(0.33_{-0.06}^{+0.05} \mathrm{M}_{\odot} \mathrm{yr}^{-1}\right)$. We trace the total gas using a combination of the $21 \mathrm{~cm} \mathrm{~Hz} \mathrm{line} \mathrm{for} \mathrm{atomic} \mathrm{hydrogen,} \mathrm{and}$ $\mathrm{CO}(J=2-1)$ data for molecular hydrogen. We have also traced the total gas using dust masses. We study the star formation law in terms of molecular gas, total gas, and gas from dust. We perform an analysis of the star formation law on a variety of pixel scales, from $25 \operatorname{arcsec}$ to $500 \operatorname{arcsec}(100 \mathrm{pc}$ to $2 \mathrm{kpc})$. At kpc scales, we find that a linear Schmidt-type power law index is suitable for molecular gas, but the index appears to be much higher with total gas, and gas from dust. Whilst we find a strong scale dependence on the Schmidt index, the gas depletion time-scale is invariant with pixel scale.
\end{abstract}

Key words: galaxies: individual: M33-galaxies: ISM - Local Group-galaxies: star formation-infrared: galaxies - submillimetre: galaxies.

\section{INTRODUCTION}

An understanding of the processes that govern star formation within a galaxy is vital to understanding how galaxies form and evolve. Historically, studying these relationships has been limited to the scale of entire galaxies, although this has led to important relations, such as the star formation law. This law relates the star formation rate (SFR) of a galaxy to its gas content, describing how efficiently gas is transformed into stars, and thus constraining theoretical models of star formation. Schmidt (1959) originally suggested a power-law scaling relation between the volume density of SFR to a volume density of gas, i.e.

$\rho_{\mathrm{SFR}} \propto \rho_{\text {gas }}^{n}$,

and this original work by Schmidt (1959) observed a power-law index of $n=2$ for the Milky Way (MW). The first extragalactic measurements of this star formation law were that of Sanduleak (1969) and Hartwick (1971), who found a value of $n$ to be $1.84 \pm$ 0.14 in the SMC and $3.5 \pm 0.12$ in M31, respectively. However, since most observations of extragalactic objects can only average a surface density along a line of sight, more recent studies use a surface, rather than a volume density, i.e.

$$
\Sigma_{\mathrm{SFR}}=A \Sigma_{\mathrm{gas}}^{N}
$$

where $\Sigma_{\text {SFR }}$ and $\Sigma_{\text {gas }}$ are the surface densities of SFR and gas, respectively. The current form of this law was studied for a series of $\sim 100$ galaxies in the seminal work of Kennicutt (1998a), which found a very tight scaling relation, with $N \sim 1.4$; this 'KennicuttSchmidt' or KS law appears to have a similar $N$ (the so-called Schmidt, or KS index), over a wide range of redshift and environments (see e.g., Kennicutt \& Evans 2012 and references therein). Although this is, necessarily, an oversimplification of a series of complex processes, this indicates that the gas content of a galaxy is a major driver of star formation, and star formation is more efficient at higher gas densities. However, these lower resolution studies were unable to determine whether the molecular or total gas content of a galaxy is more strongly correlated with star formation (e.g. Wong \& Blitz 2002). It is also unclear from these works whether star formation is governed by local processes within star-forming clouds (e.g. Krumholz \& McKee 2005), or global processes such as cloud-cloud interactions (e.g. Wyse 1986).

One physical interpretation of this empirically derived law is that roughly constant fractions of the total gas present in molecular clouds convert into stars on their free-fall time (Elmegreen 1994; Krumholz \& Thompson 2007). This interpretation produces a Schmidt index of 1.5. Another is that the SFR is dictated by the 
amount of dense molecular gas, with the star formation law being linear given a constant dense gas fraction. In this case, the traditional superlinear star formation law is simply an artifact of the variations in this dense gas fraction between the star-forming disk galaxies and starburst galaxies used in studies (Lada et al. 2012). For a series of nearby spiral galaxies, Bigiel et al. (2008) found $N \sim 1$ to be a suitable index at sub-kpc scales when considering $\mathrm{H}_{2}$, traced by the ${ }^{12} \mathrm{CO}(J=1-0)$ line - this would indicate that the molecular gas is simply counting uniform populations of giant molecular clouds (GMCs).

Dense (with number densities, $n>10^{4} \mathrm{~cm}^{-3}$ ) molecular gas is also a promising tracer of star formation, as stars are believed to condense out of the dense gas in GMCs (André et al. 2010; Lada, Lombardi \& Alves 2010). If this is the case, then we would expect the dense gas mass and SFR to be strongly and linearly correlated. A number of works comparing the far-infrared (FIR) luminosity and proxies for this dense gas, such as $\mathrm{HCN}$ or $\mathrm{HCO}^{+}$have found this to be the case (e.g. Gao \& Solomon 2004a,b; García-Burillo et al. 2012). These relationships appear to hold down to $\sim \mathrm{kpc}$ regions (Bigiel et al. 2015; Usero et al. 2015), and large programs are looking to extend these samples (Gao et al. 2017).

With the advent of higher resolution, multiwavelength surveys of nearby galaxies, our understanding of this star formation law has improved dramatically. It has been suggested that the KS law would appear to break down on scales similar to that of a GMC $(\sim 10$ 100 pc; e.g. Onodera et al. 2010; Boquien et al. 2015; Khoperskov \& Vasiliev 2017). Bigiel et al. (2008) claim that the molecular, rather than total gas better correlates with SFR. Work by Ford et al. (2013) suggests that a superlinear $N$ is suitable for the total gas content of M31, whilst a sublinear star formation law is applicable when considering only molecular gas. Leroy et al. (2008) have found a radial dependence in $N$, with decreasing star formation efficiency at larger galactocentric radius for a series of 23 nearby galaxies. For apertures targeted on $\mathrm{CO}$ and $\mathrm{H} \alpha$ peaks in M33, Schruba et al. (2010) find a breakdown at the star formation law at scales of $300 \mathrm{pc}$, although with increasing aperture size the correlation is restored, which they argue indicates variations between the evolutionary states of GMCs in a galaxy.

M33 is the third massive disk galaxy of our Local Group (behind the MW and M31), and is an excellent laboratory for high-resolution extragalactic studies. M33 is a late-type spiral galaxy located at a distance of $840 \mathrm{kpc}$ (Madore \& Freedman 1991), and is more faceon than M31 with a moderate inclination of $56^{\circ}$ (Regan \& Vogel 1994). With a large optical extent $\left(R_{25}\right)$ of $30.8 \operatorname{arcmin}(\sim 7.4 \mathrm{kpc}$, Paturel et al. 2003), M33 is ideally suited for detailed study. Despite being smaller and less massive than the MW, it has a much higher gas fraction, and is actively star forming throughout its disk (Heyer et al. 2004). It has a high star formation efficiency (Gardan et al. 2007), with a molecular gas depletion time-scale of 1.6-3.2 × $10^{8} \mathrm{yr}$, shorter than other local spiral galaxies ( 1-3 Gyr, Kennicutt 1998b; Murgia et al. 2002; Wong \& Blitz 2002). It also has a roughly half-solar metallicity $(12+\log (\mathrm{O} / \mathrm{H})=8.36 \pm 0.04$; Rosolowsky \& Simon 2008), with a shallow metallicity gradient, making it more analogous to younger or higher redshift galaxies than the MW or M31. Recent work has suggested that following a tidal encounter with M31 (McConnachie et al. 2010; Wolfe et al. 2013), the stripped gas now returning to the disk of M33 is fuelling star formation (Putman et al. 2009). Despite this, the disk of M33 is relatively unperturbed. This is in contrast to the Magellanic Clouds, which are highly disturbed, irregular dwarf galaxies. It is, therefore, particularly noteworthy to study the interplay between the gas and star formation of M33.
The choice of SFR tracer is critical, as they are sensitive to different time-scales and stellar populations. For instance, Verley et al. (2007) found a SFR of $0.2 \mathrm{M}_{\odot} \mathrm{yr}^{-1}$ in M33 when considering IR data from Spitzer, whilst $\mathrm{H} \alpha$ and UV gives a much higher SFR of $0.45 \pm 0.10 \mathrm{M}_{\odot} \mathrm{yr}^{-1}$ (Verley et al. 2009). Similarly, the adopted tracer of gas and fitting method can have a large impact on the calculated $N$. For M33, Heyer et al. (2004) find a molecular Schmidt index, $N$ of $1.36 \pm 0.08$, but Verley et al. (2010) find a range of indices $(1.0<N<2.6)$, depending on the gas tracer and fitting method employed.

A fundamental assumption of the method used to trace SFR is that the emission arises either directly from young stars, or from their heating of the ISM. UV emission directly tracers the unobscured star formation from these young stars, and hence is a good tracer of this recent star formation. However, UV is particularly sensitive to dust attenuation, and so in this work we combine this with $24 \mu \mathrm{m}$ emission to account for re-emission of this dust-absorbed light.

Another estimate of SFR is the total infrared (TIR) luminosity. This prescription assumes that the dust is heated entirely by young stars. This measure of SFR, however, will miss the starlight that is not absorbed by the dust (e.g. Hirashita et al. 2001), and therefore underestimate the SFR. Older stellar populations will also contribute to the heating of this dust, potentially causing an overestimate of the SFR (e.g. Cortese et al. 2008).

Finally, we use a third measure of the SFR - the spectral energy distribution (SED) fitting tool Multi-wavelength Analysis of Galaxy Physical Properties (MAgPhys; da Cunha, Charlot \& Elbaz 2008). This tool fits an SED from a library of models to a series of provided fluxes, and outputs the physical parameters of the fitted model. MAGPHYS allows for a bursty star formation history, and variations in SFR down to $1 \mathrm{Myr}$. This is particularly important at sub-kpc scales, where the assumption of stationary star formation may be inappropriate (Relaño \& Kennicutt 2009). It also includes an energy balance between the stellar and dust components of the SED, accounts for filter response, and performs a thorough Bayesian error analysis.

The gas present in the ISM of a galaxy is dominated by hydrogen, both in its atomic $(\mathrm{HI})$ and molecular $\left(\mathrm{H}_{2}\right)$ phases. Whilst the $\mathrm{HI}$ can be measured directly, via the $21 \mathrm{~cm}$ line, $\mathrm{H}_{2}$ cannot be traced directly as it is has a low mass, and lacks a dipole moment. Hence, the next most abundant molecule, $\mathrm{CO}$, is commonly used as a proxy, and is traced via its rotational transitions - in the case of our work, the $\mathrm{CO}(J=2-1)$ line.

Alternatively, the gas content of a galaxy can, theoretically, be traced by the cold dust continuum (e.g. Hildebrand 1983; Eales et al. 2012; Magdis et al. 2012). However, the resolution of these dust maps have usually been limited by the resolution of the Herschel (Pilbratt et al. 2010) Space Observatory. This is particularly true of objects with large angular extents, as ground-based instruments such as SCUBA-2 are poor at recovering large-scale structure due to atmospheric effects. We have developed a technique to combine higher resolution SCUBA-2 data with the larger spatial frequency data of other instruments (Smith et al. in preparation), and we present those data for the first time in this work. Using this, we can sample the dust continuum from 100 to $850 \mu \mathrm{m}$ at an unprecedented spatial scale of $100 \mathrm{pc}$, a factor of $\sim 1.4$ better than previous panchromatic galaxy studies (Viaene et al. 2014). This allows us to probe the relationships between star formation and constituents of the ISM at the scales of individual star-forming regions.

The layout of this paper is as follows: we present an overview of the data used to calculate the SFR, as well as the data processing 
techniques required to carry out a pixel-by-pixel study of the star formation law (Section 2) as well as our methods of calculating SFR at these small scales, and comparisons between them (Section 3). We present an overview of our various methods of tracing the gas within M33 (Section 4). We then use this data to study the star formation law (Section 5), before our discussion and main conclusions (Section 6).

\section{DATA}

In this section, we present an overview of the datasets used in order to probe the star formation in M33. Particularly for MAGPHYs, it is important to sample the entire galaxy SED from UV to submillimetre (sub-mm), so as to provide meaningful constraints on its output parameters. Examples of the images used in this work are shown in Fig. 1.

\subsection{Submillimetre and IR data}

Sub-mm data of M33 at 450 and $850 \mu \mathrm{m}$ was obtained with SCUBA2 (Holland et al. 2013) on the James Clerk Maxwell Telescope (JCMT) between 2017 October 17 and 2017 November 21 under Program ID M17BP003. These 30 arcmin PONG maps were taken in band 1 weather (with $225 \mathrm{GHz}$ opacity, $\tau_{225}<0.05$ ). This data has a resolution of $8 \operatorname{arcsec}$ at $450 \mu \mathrm{m}$, and $14 \operatorname{arcsec}$ at $850 \mu \mathrm{m}$. We have also included earlier, public data taken between 2012 July 1 and 2012 July 12 under Program ID M12AC16, taken in marginal band 2 /band 3 weather $\left(0.06<\tau_{225}<0.12\right)$ and acquired from the CADC archive. ${ }^{1}$ The final maps have an RMS noise level of $33 \mathrm{mJy}$ beam $^{-1}(450 \mu \mathrm{m})$ and $4 \mathrm{mJy} \mathrm{beam}^{-1}(850 \mu \mathrm{m})$. SCUBA-2 data is unreliable at recovering low spatial frequencies due to the data reduction process due to atmospheric and instrumental variations requiring large-scale filtering in the data reduction process. To mitigate for this, we have developed a technique to combine the high spatial frequencies of these data with low spatial frequencies from other telescopes, so as to increase the resolution without losing large-scale structure. For the $450 \mu \mathrm{m}$ data, we set a maximum filter scale of 120 arcsec in the data reduction pipeline. The large-scale structure is recovered using data from the Herschel Spectral and Photometric Imaging Receiver (SPIRE; Griffin et al. 2010) instrument, at $500 \mu \mathrm{m}$, adjusting the flux accordingly (multiplying the flux by a factor of 1.524, which assumes a fixed dust emissivity in$\operatorname{dex}, \beta$, of 2). The combination of these maps was performed using the FEATHER task in the Common Astronomy Software Applications (CASA) package. ${ }^{2}$ The SCUBA-2 $850 \mu \mathrm{m}$ map was treated in much the same way, but instead using $353 \mathrm{GHz}$ Planck data, and using a maximum filter scale of 320 arcsec. The maps cover an area larger than 30 arcmin, but the radial dependence on the noise was found to cause artifacts in the feathering process. Hence, for these maps we only use the central 30 arcmin.

Complementing this sub-mm data, we made use of the other two SPIRE bands, at 250 and $350 \mu \mathrm{m}$, with a resolution of 18 and 25 arcsec, respectively. This SPIRE dataset was obtained as part of the Herschel M33 extended survey (HerM33es; Kramer et al. 2010) open time key project. This project mapped the entirety of M33 with SPIRE, covering a total area of the sky of approximately 70 arcmin $\times 70$ arcmin. This data was obtained from the Herschel Science

\footnotetext{
${ }^{1}$ http://www.cadc-ccda.hia-iha.nrc-cnrc.gc.ca/en/

2 https://casa.nrao.edu/
}

Archive, ${ }^{3}$ utilizing the Standard Product Generation (SPG) software v14.1.0. These maps have been calibrated for extended sources, and we include the small beam correction recommended in the SPIRE handbook ${ }^{4}$ The RMS noise levels of this SPIRE data are 14.1, 9.2, and $8 \mathrm{mJy}_{\text {beam }}^{-1}$ at 250,350 , and $500 \mu \mathrm{m}$, respectively.

The HerM33es project also mapped this same 70 arcmin $\times 70$ arcmin at 100 and $160 \mu \mathrm{m}$ with the Photoconductor Array Camera and Spectrometer (PACS; Poglitsch et al. 2010). This data has a resolution of 7.7 and 12 arcsec, respectively, with an RMS noise level of $2.6 \mathrm{mJy} \mathrm{pixel}^{-1}(100 \mu \mathrm{m})$ and $6.9 \mathrm{mJy} \mathrm{pixel}^{-1}(160 \mu \mathrm{m})$. This data was processed using the SPG software v14.2.0, using the JSCANAM map-maker. Again, these maps are calibrated for extended sources.

Our first source of near-infrared (NIR) data is from the Widefield Infrared Survey Explorer (WISE; ${ }^{5}$ Wright et al. 2010). These images have wavelengths of $3.4,4.6,12$, and $22 \mu \mathrm{m}$ with FWHM of $6.1,6.4,6.5$, and 12 arcsec, respectively. We used MONTAGE ${ }^{6}$ to mosaic together various frames from the AllWISE data release, incorporating both the WISE cryogenic and NEOWISE (Mainzer et al. 2011) post-cryogenic surveys. MONTAGE also matches background levels between each frame, so that overlaps between frames match as closely as possible. However, this is not a background subtraction, so to allow us to adequately model the sky, we created a $3 \mathrm{deg}^{2}$ mosaic of M33 in each band, to ensure we had a sufficiently large amount of sky to model.

Additional IR data was obtained by the Spitzer Infrared Array Camera (IRAC; Fazio et al. 2004), as part of the Local Volume Legacy (LVL; Dale et al. 2009) Survey. ${ }^{7}$ We used data from IRAC taken at 3.6, 4.5, 5.8, and $8 \mu \mathrm{m}$. The resolution of these data are $\sim 2$ arcsec, and cover approximately $90 \operatorname{arcmin} \times 60$ arcmin. Along with IRAC data, we also made use of the Multiband Imaging Photometer for Spitzer (MIPS; Rieke et al. 2004) data, taken again as part of the LVL. This data covers approximately 130 arcmin $\times 80$ arcmin, and is at 24 and $70 \mu \mathrm{m}$, with a resolution of 6 and 18 arcsec, respectively. The overlap between several Spitzer and WISE bands improves sampling of the mid-infrared (MIR) SED, reducing the dependence of the fit on a single point.

\subsection{UV and optical data}

The UV data used in this work comes from the Galaxy Evolution Explorer (GALEX; Martin et al. 2005), obtained by Thilker et al. (2005). Data was obtained for both the FUV (1516 $\AA$ ) and NUV

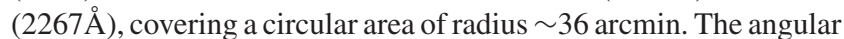
resolution of this data is $4.2 \operatorname{arcsec}$ and $5.3 \operatorname{arcsec}$ for the FUV and NUV, respectively, and with $\sim 3 \mathrm{ks}$ exposures, typical $1 \sigma$ RMS flux sensitivities are $\sim 28 \mathrm{AB}$ mag $\operatorname{arcsec}^{-2}$.

The optical data used in this study comes from the Sloan Digital Sky Survey ( SDSS ${ }^{8}$ York et al. 2000). Using only primary frames from the SDSS DR13 (Alam et al. 2015), a mosaic of 3deg ${ }^{2}$ was created, allowing enough sky to accurately model the background. The SDSS data was mosaicked together using MONTAGE for all five

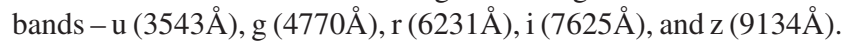

\footnotetext{
${ }^{3} \mathrm{http} / / /$ archives.esac.esa.int/hsa/whsa

${ }^{4}$ http://herschel.esac.esa.int/Docs/SPIRE/spire_handbook.pdf

${ }^{5} \mathrm{http} / / /$ irsa.ipac.caltech.edu/Missions/wise.html

${ }^{6} \mathrm{http}: / /$ montage.ipac.caltech.edu

${ }^{7}$ http://irsa.ipac.caltech.edu/data/SPITZER/LVL/

${ }^{8}$ https://dr13.sdss.org/home
} 


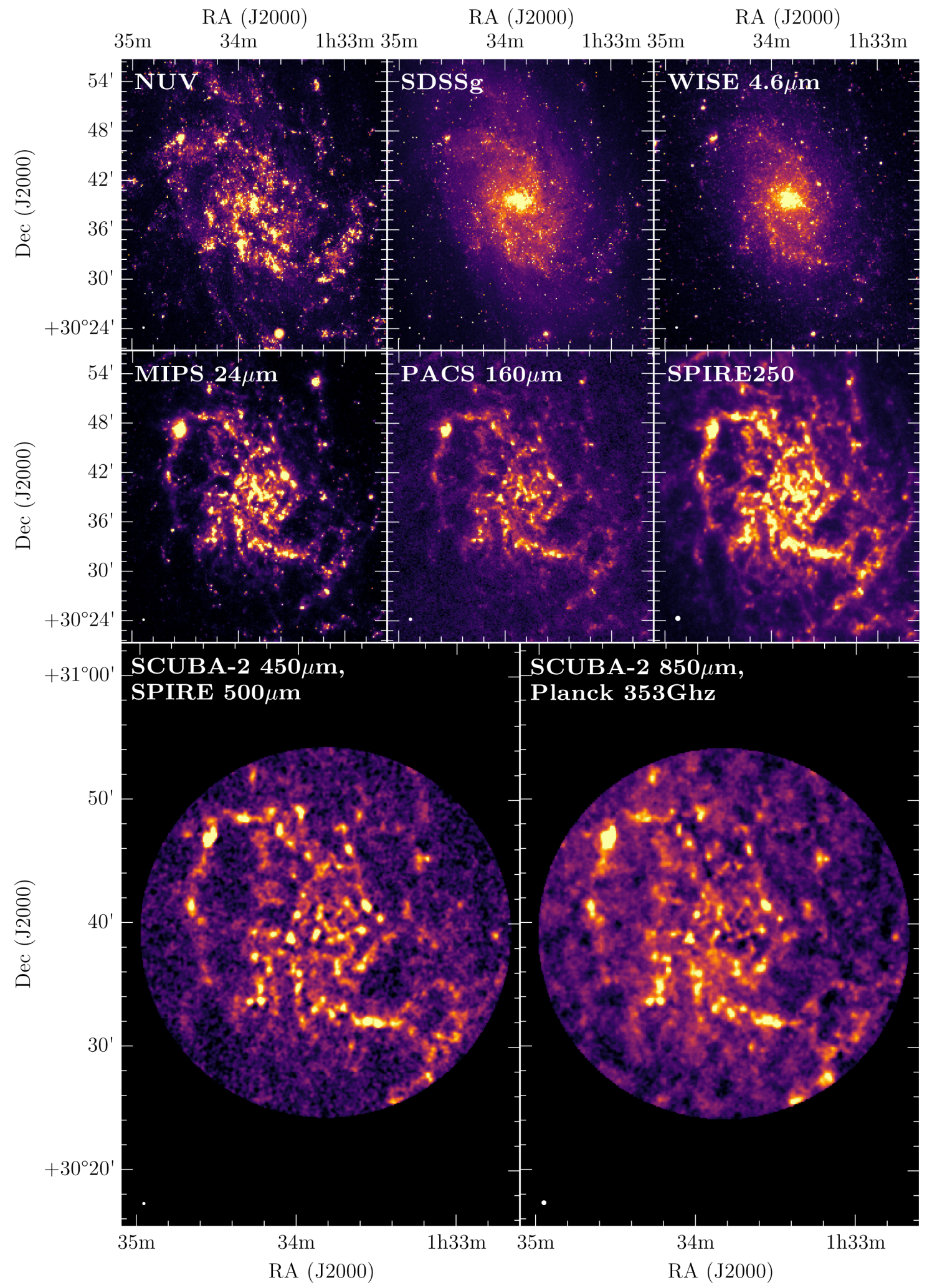

Figure 1. Examples of the data used to calculate SFR in this work. From top left: GALEX NUV (Lee et al. 2011) map, SDSS g band mosaic, WISE $4.6 \mu \mathrm{m}$ mosaic, MIPS $24 \mu \mathrm{m}$ (Dale et al. 2009) map, PACS $160 \mu \mathrm{m}$ and SPIRE $250 \mu \mathrm{m}$ (Kramer et al. 2010) data, and SCUBA-2 data $-450 \mu \mathrm{m}$ combined with SPIRE $500 \mu \mathrm{m}$, and $850 \mu \mathrm{m}$ combined with Planck $353 \mathrm{GHz}$ data. The SCUBA-2 maps have both been convolved with a 6 arcsec Gaussian and cropped to circles of 15 arcmin radius, to aid visualisation. The beam for each frame is shown as a solid circle in the bottom left - in many cases, the beam is negligible compared to the image size. 


\subsection{Data preparation}

Incorporating data from a variety of sources requires careful consideration so that meaningful comparisons can be drawn on a pixelby-pixel basis. Hence, it was necessary to process the dataset, so as to make it homogeneous, and we give a description of that process here.

\subsubsection{Background subtraction}

For each frame, we performed a background subtraction. Depending on the background, we employed a variety of methods to achieve this. Before this background subtraction process, we also converted all of the data into units of $\mathrm{Jy} / \mathrm{px}$, if it was required.

GALEX: The average background for the GALEX frame was found to be 0 , with no clear gradient, so no background subtraction was applied.

SDSS: Due to the mosaicking process, the SDSS frames had a varying non-zero background. In order to remove this background, M33 was masked (using an ellipse of $80 \operatorname{arcmin} \times 60$ arcmin) before fitting and subtracting a $2 \mathrm{D}$ polynomial. This reduced the background variation in the image to the order of a few percent, consistent with, e.g. Corbelli et al. (2014).

Spitzer, WISE, Herschel : For the Spitzer, WISE, and Herschel frames, the background was constant throughout the image, so in these frames a median background was subtracted using a $3 \sigma$ clipped median after masking all sources with a signal to noise $(\mathrm{S} / \mathrm{N})>2$.

SCUBA-2: The SCUBA-2 data reduction process performs an iterative sky modelling and subtraction procedure (Chapin et al. 2013), so no further sky subtraction was performed.

Following background subtraction, we apply a Galactic extinction correction for frames with central wavelengths shorter than $4.6 \mu \mathrm{m}$ (the WISE-2 band). We use the method prescribed by Schlafly \& Finkbeiner (2011) for the central position of M33, provided by the IRSA DUST ${ }^{9}$ service. Due to the large angular extent of M33, the extinction correction can vary by a significant amount (14 per cent in the FUV and NUV frames, for example). We included this variation in our uncertainty treatment (Section 2.4).

\subsubsection{Star masking}

It was also necessary to mask any flux contamination from foreground stars. We masked stars using a comparison of the UV fluxes, as described by Leroy et al. (2008). Using SEXTRACTOR (Bertin \& Arnouts 1996), we found the positions of all $5 \sigma$ detections in the NUV. An optimal aperture size was then calculated for each of these detections using a similar method to Viaene et al. (2014), by calculating a radius for each detection where the flux at that radius dropped below two times the local background level. These apertures were then placed in the FUV maps and the flux within each calculated. Leroy et al. (2008) found that foreground stars have an NUV-to-FUV flux ratio of $\gtrsim 15 \pm 5$. Upon visual inspection, we found that a ratio 15 was insufficient to mask all foreground stars, and so opted instead for a value of 10 . These stars were subsquently masked in all frames up to $\sim 20 \mu \mathrm{m}$, after which the foreground star emission was no longer an issue. Of the $\sim 6000$ sources detected with SEXTRACTOR, around 200 were masked.
Table 1. Calibration uncertainty for each pixel.

\begin{tabular}{lcc}
\hline Telescope & Calibration Uncertainty & Reference \\
\hline GALEX FUV & $5 \%$ & 1 \\
GALEX NUV & $3 \%$ & 1 \\
SDSS u & $2 \%$ & 2 \\
SDSS g,r,i,z & $1 \%$ & 2 \\
IRAC-1 & $10 \%$ & 3 \\
IRAC-2 & $10 \%$ & 3 \\
IRAC-3 & $15 \%$ & 3 \\
IRAC-4 & $15 \%$ & 3 \\
MIPS $24 \mu \mathrm{m}$ & $4 \%$ & 4 \\
MIPS 70 $\mu \mathrm{m}$ & $5 \%$ & 5 \\
WISE W1 & $2.4 \%$ & 6 \\
WISE W2 & $2.8 \%$ & 6 \\
WISE W3 & $4.5 \%$ & 6 \\
WISE W4 & $5.7 \%$ & 6 \\
PACS & $5 \%$ & 7 \\
SPIRE & $5.5 \%$ & 8 \\
SCUBA-2 $450 \mu \mathrm{m}$ & $12 \%$ & 9 \\
SCUBA-2 $850 \mu \mathrm{m}$ & $8 \%$ & 9 \\
Planck $353 \mathrm{GHz}$ & $3 \%$ & 10
\end{tabular}

Note: References: (1) Morrissey et al. (2007); (2) Padmanabhan et al. (2008); (3) Dale et al. (2009); (4) Engelbracht et al. (2007); (5) Gordon et al. (2007); (6) Jarrett et al. (2011) (7) PACS Handbook ${ }^{9}$; (8) SPIRE Handbook; (9) Dempsey et al. (2013); (10) Planck Collaboration XVI (2014)

\subsubsection{Convolution and regridding}

In order to make comparisons on a pixel-by-pixel basis, it was necessary to match the data to a common resolution and pixel scale. In order to do this, we made use of the convolution kernels ${ }^{10}$ provided by Aniano et al. (2011) in order to achieve a common resolution. In this case, we match everything to the PSF of the SPIRE $350 \mu \mathrm{m}$ data, which has a FWHM of 25 arcsec. We regridded all of the data to a common pixel scale of 25 arcsec (corresponding to a spatial scale of $100 \mathrm{pc}$ ), which ensured that we could safely assume each pixel to be statistically independent. For an ellipse of $60 \mathrm{arcmin} \times 70 \mathrm{arcmin}$, this corresponded to 19004 pixels.

\subsection{Uncertainties}

There were a number of uncertainties to take into account for each pixel. In areas of high $\mathrm{S} / \mathrm{N}$, the calibration error $\left(\sigma_{\text {cal }}\right)$ of the instrument dominates. We considered these calibration errors, which are summarised in Table 1. In the case of the SCUBA-2 maps combined with other, lower resolution maps, we take the calibration error as the sum of the two relevant uncertainties in quadrature. We also included uncertainties from the varying Galactic extinction correction $\left(\sigma_{\text {ext }}\right)$ due to the large angular extent of M33. The GALEX data is most affected by this, with a scatter of 14 per cent. We also considered the background variation $\left(\sigma_{\mathrm{bg}}\right)$ in each frame. For this, we took the standard deviation of the background, having masked any sources greater than $2 \sigma$. In the case of the SDSS frames, this error also incorporated any remaining large-scale residuals due to the mosaicking process. Finally, for the GALEX and SDSS frames an uncertainty arose from the small numbers of photons incident at these wavelengths $\left(\sigma_{\text {poiss }}\right)$. These errors are Poissonian. To estimate these errors, the flux was converted back into a count number and the square root of this count converted into a flux to give an error.

\footnotetext{
${ }^{10}$ http://www.astro.princeton.edu/ $\sim$ ganiano/Kernels.html
} 
The total uncertainty for each pixel was given by

$\sigma_{\text {total }}=\sqrt{\sigma_{\text {cal }}^{2}+\sigma_{\text {bg }}^{2}+\sigma_{\text {ext }}^{2}+\sigma_{\text {poiss }}^{2}}$

\section{CALCULATING SFR}

Different tracers of SFR are subject to different systematic effects, such as dust attenuation and the impact of older stellar populations. To ensure that our pixel-by-pixel SFR measurements are robust, we have compared three different SFR calibrations.

\subsection{Total infrared luminosity}

We first calculated the SFR from the integrated TIR luminosity. TIR luminosity effectively captures the bolometric luminosity of the dust-obscured stellar population, and so traces the starlight absorbed by the dust in a galaxy. Two important assumptions for TIR luminosity tracing the total SFR of a galaxy are that all of the emission from the young stellar population is absorbed by dust, and that the dust heating is exclusively from these young stars. Both of these assumptions are oversimplifications for galaxies (see e.g., Hirashita et al. 2001; Cortese et al. 2008), and whilst on the scale of integrated galaxies appear to balance each other out (e.g. Kewley et al. 2002), on a pixel-by-pixel basis these assumptions may not hold true. However, the TIR SFR is a useful diagnostic, and informs us both about the stellar population of a galaxy, as well as its dust content. In this work, we have used the calibration given by Kennicutt \& Evans (2012), using values from Hao et al. (2011) and Murphy et al. (2011), integrating the greybody fit from 3 to $1100 \mu \mathrm{m}$ :

$$
\log _{10}\left(\mathrm{SFR}_{\mathrm{TIR}}\right)=\log _{10}\left(L_{\mathrm{TIR}}\right)-43.41 \text {, }
$$

where $L_{\mathrm{TIR}}$ is in ergs s${ }^{-1}$. This assumes the default IMF of Starburst99 (Leitherer et al. 1999), the broken Kroupa (2001) power law with a maximum mass of $120 \mathrm{M}_{\odot}$, and a time-scale of $\sim 100 \mathrm{Myr}$. Including an error of 30 per cent to estimate an uncertainty in the IMF and amount of dust attenuation, we found a TIR SFR of 0.17 $\pm 0.06 \mathrm{M}_{\odot} \mathrm{yr}^{-1} \cdot{ }^{11}$

\section{2 $\mathrm{FUV}+24 \mu \mathrm{m}$}

The TIR SFR misses the unattenuated component of the SFR, which can vary dramatically in different environments. Particularly significantly for M33, the unattenuated component can be a major proportion of the total SFR in low-metallicity environments (e.g. Hirashita et al. 2001). One effective method of overcoming this is to combine IR measurements tracing obscured star formation with UV emission that measures the unattenuated star formation. In this work, we have elected to combine the GALEX FUV and MIPS $24 \mu \mathrm{m}$ data. FUV emission traces unobscured star formation over a time-scale of 10-100 Myr (e.g. Kennicutt 1998a; Calzetti et al. 2005), whilst the $24 \mu \mathrm{m}$ emission traces emission from small dust grains heated by starlight over a time-scale of $\sim 10 \mathrm{Myr}$ (e.g. Calzetti et al. 2005, 2007). We use the SFR density prescription of Leroy et al. (2008):

$\Sigma_{\mathrm{SFR}}=8.1 \times 10^{-2} I_{\mathrm{FUV}}+3.2_{-0.7}^{+1.2} \times 10^{-3} I_{24}$,

where $\Sigma_{\mathrm{SFR}}$ is in units of $\mathrm{M}_{\odot} \mathrm{kpc}^{-2} \mathrm{yr}^{-1}, I_{\mathrm{FUV}}$ and $I_{24}$ are intensities in units of MJy/sr. Again, we have assumed the default Starburst99 settings, and a time-scale of $100 \mathrm{Myr}$. Some of the emission at these

\footnotetext{
${ }^{11}$ http://herschel.esac.esa.int/Docs/PACS/pdf/pacs_om.pdf
}

wavelengths may be due to an older stellar population (e.g. Kennicutt et al. 2009), indicated by a correlation between these bands and the $3.6 \mu \mathrm{m}$ data. To correct for this, we remove this contribution using

$I_{\mathrm{FUV}, \text { corr }}=I_{\mathrm{FUV}}-\alpha_{\mathrm{FUV}} I_{3.6}$,

$I_{24, \text { corr }}=I_{24}-\alpha_{24} I_{3.6}$,

where $\alpha_{\mathrm{FUV}}=3 \times 10^{-3}$ and $\alpha_{24}=0.1$ (Leroy et al. 2008). This correction has the effect of reducing the total SFR by $0.01 \mathrm{M}_{\odot} \mathrm{yr}^{-1}$. Using this method, we find a total SFR of $0.25_{-0.07}^{+0.10} \mathrm{M}_{\odot} \mathrm{yr}^{-1}$, somewhat higher, but still consistent with the TIR SFR.

\subsection{MAGPHYS}

Finally, we calculate the SFR using MAGPHYS. MAGPHYS fits an SED from a large library of optical and IR models with known, physically motivated input parameters. It finds the best fit to the data in each case, and outputs the physical parameters of these fits, as well as modelling uncertainties upon them. For the optical models, MAGPHYs assumes a Chabrier (2003) initial mass function (IMF), which it evolves using the Bruzual \& Charlot (2003) stellar population synthesis (SPS) model, and has a star formation history (SFH) resolution of 1 Myr. Dust obscuration is calculated using the model of Charlot \& Fall (2000). The total MAGPHYS SFR as calculated from the integrated flux across the galaxy is $0.33_{-0.06}^{+0.05} \mathrm{M}_{\odot} \mathrm{yr}^{-1}$. These uncertainties only take into account uncertainties on the flux, so are likely an underestimate of the true error. This value for SFR is consistent with work by Verley et al. (2009), but higher than both the total SFR calculated using FUV+24 $\mu \mathrm{m}$ and from TIR luminosity. However, whilst MAGPHYs has a parameter space suitable for integrated galaxies, a single pixel in M33 is far outside this space in terms of flux. We artificially increased each flux by a factor of $10^{4}$ to put it within MAGPHYs parameter space. Most quantities from MAGPHYS tend to be ratios and so are not affected by this scaling the four that scale with flux do so linearly, and are the SFR, dust mass $\left(M_{\text {dust }}\right)$, stellar mass $\left(M_{\text {star }}\right)$, and dust luminosity $\left(L_{\text {dust }}\right)$.

One important feature of MAGPHYs is that it enforces an energy balance, where all attenuated light is re-emitted by the dust. Whilst this may hold true for a whole galaxy, the light from neighbouring regions may have an impact on the pixel in question in these sub-kpc regions. If this is the case, there may be an offset between the values calculated on a per-pixel basis, and those on a global scale. To test this, we also calculated the SFR from the sum of the individual pixels, giving us a value of $0.33 \pm 0.10 \mathrm{M}_{\odot} \mathrm{yr}^{1}$. This indicates that ${ }^{-}$MAGPHYs is suitable for pixel-by-pixel fitting, despite its original intent for galaxy-scale SED fits.

A benefit of using MAGPHYS is that the entire range of data can be used, regardless of the errors on each individual point. The fitted parameters can then be filtered a posteriori, based on how well constrained they are. MAGPHYS gives a probability distribution function (PDF) for each parameter, and the width of this PDF indicates how well constrained each parameter is. MAGPHYs provides in its outputs the 2.5 th, 16th, 50th, 84th, and 97.5 th percentiles for each parameter, we calculated a relative uncertainty for each pixel - any pixels which did not satisfy

$0.5 \times \frac{p_{86}-p_{16}}{p_{50}}<0.32$

where $p_{x}$ is the $x$ th percentile, were removed. This method of pixel filtering has previously been employed by Viaene et al. (2014), who found it a sufficient cut to remove any broad or bimodal (i.e. poorly 
constrained) PDFs. We have used this method of filtering pixels in the proceeding analysis, rather than a more traditional S/N cut on the map, leaving us with 6574 pixels. In this work, rather than using the SFR for the best fit model, we used the median-likelihood estimate given by the PDF. Some example SEDs, as well as the global SED can be seen in Fig. 2.

\subsection{SFR comparisons}

We find that the lowest SFR calculated is from the TIR luminosity, at $0.17 \pm 0.06 \mathrm{M}_{\odot} \mathrm{yr}^{-1}$. Including the unattenuated component of the starlight increases the SFR to $0.25_{-0.07}^{+0.10} \mathrm{M}_{\odot} \mathrm{yr}^{-1}$ using FUV+24 $\mu \mathrm{m}$, and even higher from MAGPHYs $\left(0.33_{-0.06}^{+0.05} \mathrm{M}_{\odot} \mathrm{yr}^{-1}\right)$. This lack of dust attenuation is highlighted in Fig. 2 - the stellar component dominates over the dust, as highlighted by the small offset between the unattenuated and attenuated lines, especially within the nucleus. That this unattenuated component accounts for $\sim 50$ per cent of the SFR highlights the importance of the inclusion of the unattenuated component, particularly in low-metallicity or dust-poor galaxies.

As well as a global SFR, we have also calculated the SFR on a perpixel basis, and these maps can be seen in Fig. 3, with a comparison of these three tracers of SFR in Fig. 4. In the cases of the TIR and FUV+24 $\mu \mathrm{m}$ SFR map, we compare only the pixels with S/N $>5 \sigma$. The three broadly agree - the RMS scatter $(\sigma)$ and median offset from the $1-1$ relation $[\Delta \log (\mathrm{SFR})]$ is summarised in Table2. However, there is a population of pixels with higher SFRs given by MAGPHYS than the other two tracers, and this is reflected in a much higher scatter. In the cases where the MAGPHYS SFR is significantly higher, this is due to MAGPHYS injecting a recent starburst. Both the SFR from TIR luminosity and FUV $+24 \mu \mathrm{m}$ assume continuous star formation over the last $100 \mathrm{Myr}$. At sub-kpc resolutions, star formation may vary over time-scales of a few Myr (Boselli et al. 2009). We find that these areas of bursty star formation tend to be associated with HII regions and stellar associations (a sample of which are shown in the rightmost panel of Fig. 3). As the spectra of HII regions strongly resemble those of starburst galaxies (e.g. Ho, Filippenko \& Sargent 1997), it is not surprising that MAGPHYs has treated them as such. Additionally, UV and FUV spectroscopy have shown that at least two populations of stars exist within the nucleus of M33, and that star formation occurred within the nucleus $\sim 40 \mathrm{Myr}$ ago (Long, Charles \& Dubus 2002), so this injected starburst within the nucleus of M33 is believable. Overall, these tracers of SFR show very similar characteristics on a pixel-by-pixel level, but as MAGPHYS uses all available data, provides an energy balance, and can take into account recent bursts of star formation, we elect to use this tracer going forwards.

\section{GAS}

\subsection{Atomic gas}

For studying the atomic hydrogen, archival $\mathrm{VLA}^{12} \mathrm{~B}, \mathrm{C}$, and D array data for the $21 \mathrm{~cm}$ line was reduced by Gratier et al. (2010). The data used was an integrated intensity map in units of $\mathrm{K} \mathrm{km} \mathrm{s}^{-1}$, with a spatial resolution of $12 \operatorname{arcsec}(\sim 50 \mathrm{pc}$ at the distance of M33). The original data cube has a spectral resolution of $1.27 \mathrm{~km} \mathrm{~s}^{-1}$. This data can be seen in the left-hand panel of Fig. 5. From the Hr $21 \mathrm{~cm}$ line a density could be immediately calculated, using the equation

12 https://science.nrao.edu/facilities/vla/archive/index presented in Rohlfs \& Wilson (1996):

$$
\Sigma_{\mathrm{HI}}=1.8 \times 10^{18} \mathrm{~cm}^{-2} /\left(\mathrm{K} \mathrm{km} \mathrm{s}^{-1}\right) \text {. }
$$

The mass of atomic gas as traced by $\mathrm{H}$ is found to be $5 \times 10^{8} \mathrm{M}_{\odot}$, including a factor of 1.36 for $\mathrm{He}$.

\subsection{Molecular gas}

$\mathrm{CO}$ is traced using the $\mathrm{CO}(J=2-1)$ rotational line data taken as part of IRAM's M33 Survey Large Program ${ }^{13}$ (Gratier et al. 2010; Druard et al. 2014), which traces the molecular gas out to a radius of $7 \mathrm{kpc}$ using IRAM's Heterodyne arRAy (HERA; Schuster et al. 2004) instrument. This data has an angular resolution of 12 arcsec and a spectral resolution of $2.6 \mathrm{~km} \mathrm{~s}^{-1}$. The integrated intensity map can be seen in the right-hand panel of Fig. 5. We use this to trace molecular hydrogen within M33, rather that the earlier $\operatorname{CO}(J=1-0)$ map (Rosolowsky et al. 2007), as this map only traces the $\mathrm{CO}$ out to a radius of $5.5 \mathrm{kpc}$, and is less sensitive $\left(\sigma_{\mathrm{RMS}}=60 \mathrm{mK}\right.$ for the $(1-0)$ map, versus $\sigma_{\mathrm{RMS}}=20 \mathrm{mK}$ for the (2-1) data).

A conversion factor must be used to convert the $\mathrm{CO}$ intensity to a number density of $\mathrm{H}_{2}\left(X_{\mathrm{CO}}\right)$, and this value is uncertain. Historically, a value of approximately $2 \times 10^{20} \mathrm{~cm}^{-2}$ (Solomon et al. 1987; Strong \& Mattox 1996; Smith et al. 2012) has commonly been used for the $\mathrm{CO}(J=1-0)$ line, as calculated for the Milky Way, but a more comprehensive study by Sandstrom et al. (2013) found an average value of $1.42 \times 10^{20} \mathrm{~cm}^{-2}$ for a sample of 26 nearby galaxies. This conversion depends on a variety of factors, including the metallicity of the galaxy in question, and can vary across a galaxy. Narayanan et al. (2012) has shown that depending on the choice of $X_{\mathrm{CO}}$, the Schmidt index can vary by \pm 0.2 and so the accurate treatment of this conversion factor is imperative. In this case, we used the values calculated by Braine et al. (2010) for M33. This work found two distinct populations: one within the central $2 \mathrm{kpc}$ of the galaxy $\left(X_{\mathrm{CO}}=1.54 \times 10^{20} \mathrm{~cm}^{-2}\right)$ and one outside this radius $\left(X_{\mathrm{CO}}=\right.$ $\left.2.87 \times 10^{20} \mathrm{~cm}^{-2}\right)$. These values are for the $\mathrm{CO}(J=1-0)$ line, so we turn these into conversion factors for the $J=2-1$ line using the commonly employed ratio of $\mathrm{CO}\left(\frac{2-1}{1-0}\right)=0.7$ (e.g. Eckart et al. 1990; Bigiel et al. 2008). Including a factor of 1.36 for $\mathrm{He}$, the total molecular gas mass was calculated to be $4.5 \times 10^{7} \mathrm{M}_{\odot}$, an order of magnitude lower than the Hi mass.

\subsection{Gas traced by dust}

We also traced the gas via the dust in a galaxy, and to that end have created two dust maps. The first was a simple one-temperature modified blackbody (MBB), with a variable $\beta$, which takes the form

$S_{v}=\frac{\kappa_{v} M_{\mathrm{dust}} B\left(v, T_{\mathrm{dust}}\right)}{D^{2}}$

where $S_{v}$ is the flux at frequency $v, \kappa_{v}$ is dust absorption coefficient at frequency $v, M_{\text {dust }}$ is the dust mass, $B\left(v, T_{\text {dust }}\right)$ is the Planck function at frequency $v$ and dust temperature $T_{\text {dust }}$, and $D$ is the distance to the source. We assumed $\kappa_{850}=0.77 \mathrm{~cm}^{2} \mathrm{~g}^{-1}$ (Dunne et al. 2000). Recent work by Clark et al. (2016) would suggest using a value of $\kappa_{500}=0.051_{-0.026}^{+0.070} \mathrm{~m}^{2} \mathrm{~kg}^{-1}$ instead, but we use this older value for consistency with MAGPHYs. We fit the MBB from 100 to $850 \mu \mathrm{m}$, using the $70 \mu \mathrm{m}$ point as an upper limit to prevent fitting to warmer dust components (Smith et al. 2010). The dust mass and $\beta$

\footnotetext{
${ }^{13} \mathrm{http} / / / \mathrm{www}$. iram.fr/ILPA/LP006/
} 


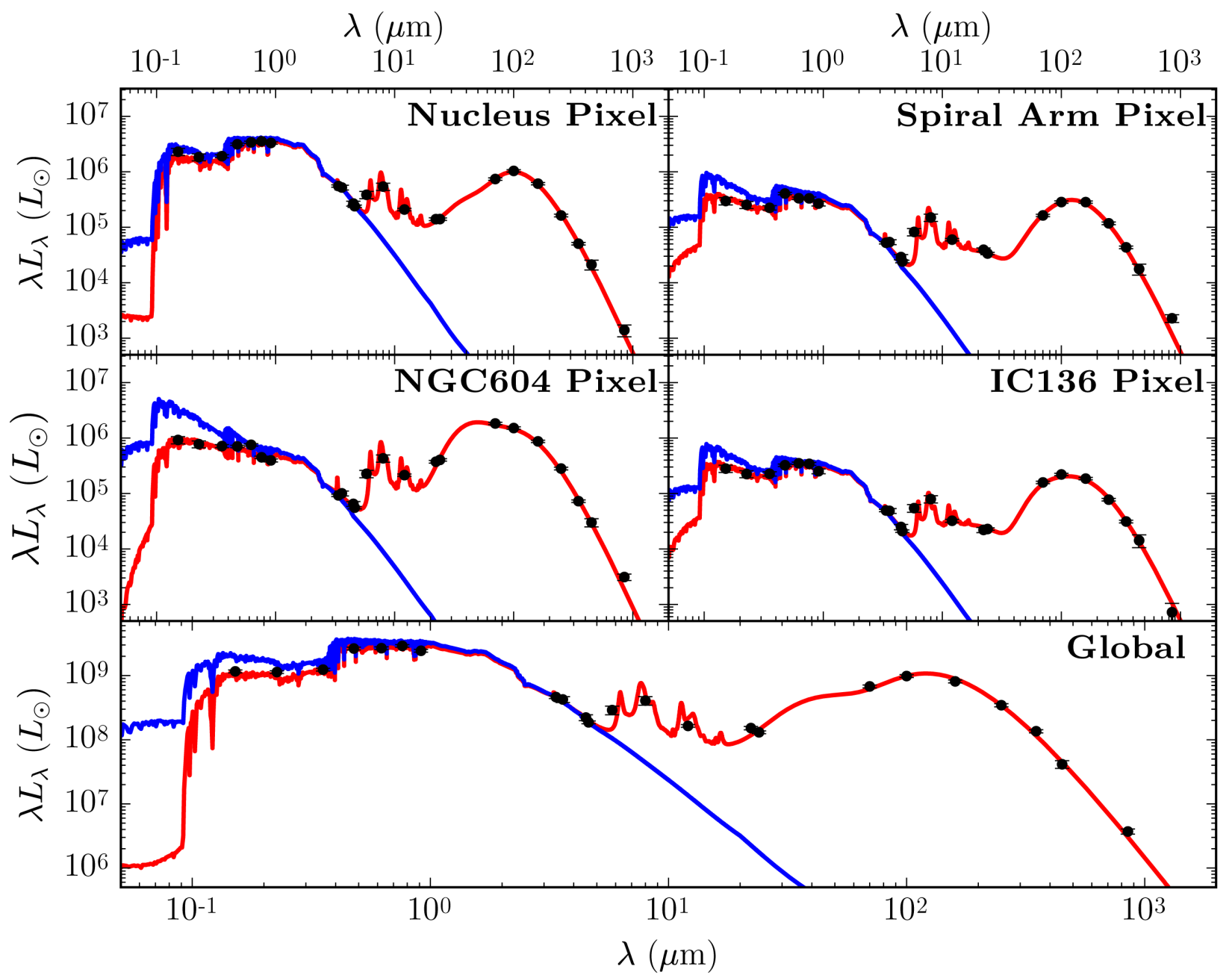

Figure 2. Example MAGPhys SEDs for single pixels. The blue line represents the unattenuated SED, the red line the best fit to the data (i.e. the dust attenuated SED). From left to right, there is a pixel from within the nucleus $(R<0.5 \mathrm{kpc})$ of M33, a pixel from the northern spiral arm, a pixel from within NGC 604 (an HII region) and a pixel from within IC136 (a stellar association). The lowermost panel shows the global SED of M33.

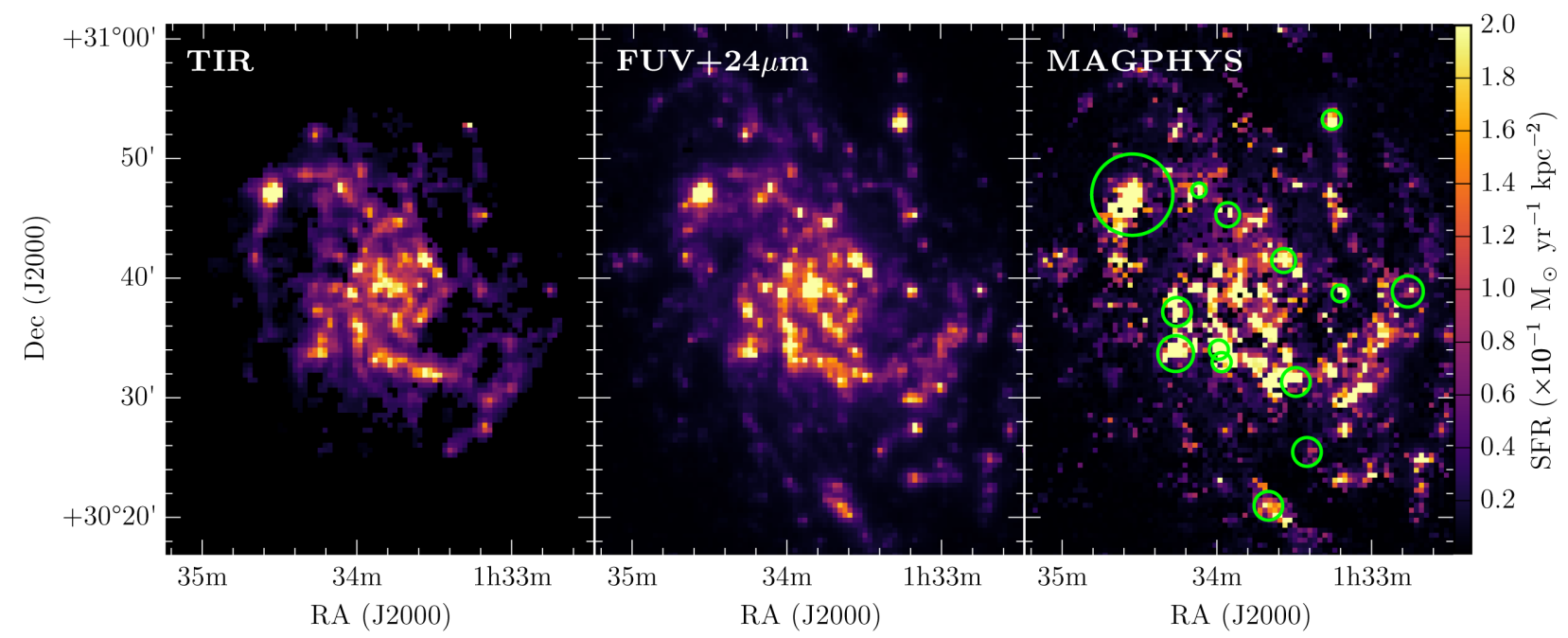

Figure 3. From left to right, SFR density maps from TIR luminosity found by integrating modified blackbody fits from 3 to $1100 \mu \mathrm{m}$, SFR from a combination of FUV $+24 \mu \mathrm{m}$ data, and median-likelihood estimates of SFR from MAGPHYS. Particularly bright pixels in the MAGPHYs map are due to recent starbursts, producing an SFR $\sim 10$ times higher than the average pixel value, and tend to be associated with HII regions and stellar clusters (a selection of which are shown as green circles). All maps have pixel sizes of 25 arcsec. 


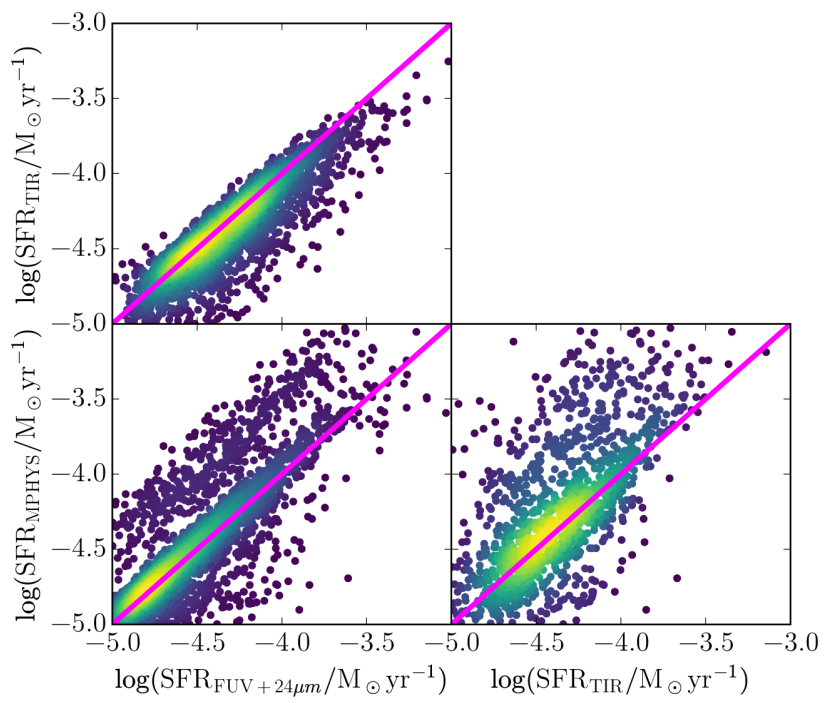

Figure 4. Comparisons for single pixel fits between left,top: SFR as calculated by the TIR luminosity and by FUV $+24 \mu \mathrm{m}$ data. Left, bottom: SFR as outputted from MAGPHYs and as calculated by a combination of FUV and $24 \mu \mathrm{m}$ data. Right, bottom: SFR parameter from MAGPHYs and calculated from the TIR luminosity. The one-to-one relation is shown as a fuchsia line. Blue points indicate a lower point density, yellow a higher point density.

Table 2. Comparisons between the three SFR tracers. For the TIR and FUV $+24 \mu$ m maps, only pixels with $\mathrm{S} / \mathrm{N}>5 \sigma$ have been considered. In the case of MAGPHYs, the filtered map is used. Given are the RMS scatter of the points $(\sigma)$ and the median offset from the $1-1$ relation $[\Delta \log (\mathrm{SFR})]$.

\begin{tabular}{lcccc}
\hline & \multicolumn{2}{c}{ SFR $_{\mathrm{FUV}+24 \mu \mathrm{m}}$} & \multicolumn{2}{c}{$\mathrm{SFR}_{\mathrm{TIR}}$} \\
& $\sigma$ & $\Delta \log (\mathrm{SFR})$ & $\sigma$ & $\Delta \log (\mathrm{SFR})$ \\
\hline SFR $_{\text {TIR }}$ & 0.16 & -0.05 & - & - \\
SFR $_{\text {MAGPHY }}$ & 0.25 & 0.04 & 0.34 & 0.08 \\
\hline
\end{tabular}

were allowed to vary freely, and the dust temperature was allowed to vary between 0 and $200 \mathrm{~K}$. This MBB fit was performed for all pixels with a $\mathrm{S} / \mathrm{N}>2.5$ in at least 5 of the Herschel/SCUBA-2 bands, giving us 2320 pixels with at least 1 degree of freedom, corresponding to a total area of $23.2 \mathrm{kpc}^{2}$. The data has filter corrections suitable for extended sources applied, although no colour corrections were accounted for. Errors were accounted for via MCMC uncertainty estimation using EMCEE. ${ }^{14}$ We used 100 walkers, each taking 300 steps, and the second half of these steps are used for error estimation to make sure the walkers have 'burnt-in'. In each case, we take the error value to be the 16th and 84th percentiles of the samples in the marginalized distributions, and the 50th percentile as our value for the quantity in question. This MCMC estimation does not take into account correlated SPIRE uncertainties, but the effects of this are expected to be minor. This dust map can be seen in the left-hand panel of Fig. 6.

The second dust map was provided as an output by MAGPHYs. The MAGPHYS dust models incorporate polycyclic aromatic hydrocarbons (PAHs), which are based on observations of M17, and these features of the SED dominate at MIR wavelengths. MAGPHYS also includes hot dust, which it models with a series of grebodies with temperatures of 850,250 , and $130 \mathrm{~K}$. The warm dust is modelled

${ }^{14} \mathrm{http} / / /$ dan.iel.fm/emcee/current/ with a modified blackbody with a emissivity index, $\beta$ of 1.5 , and can vary between 30 and $60 \mathrm{~K}$. The cold dust is modelled in much the same way, but with $\beta=2$, and can vary between 15 and $25 \mathrm{~K}$. This map can be seen in the right-hand panel of Fig. 6 .

Although these dust parameters are not unreasonable for an entire galaxy, at these sub-kpc scales some pixels may not fall within the standard MAGPHYS parameter space. We used an extended library of dust models (Viaene et al. 2014) that increase the parameter space of the cold dust temperature from $10 \mathrm{~K}<T_{\mathrm{C}}<30 \mathrm{~K}$, and the warm dust temperature to $30 \mathrm{~K}<T_{\mathrm{W}}<70 \mathrm{~K}$. As with the SFR map, we performed filtering to remove any pixels for which the cold or warm dust temperatures were poorly constrained. The distributions of the cold and warm dust for these filtered pixels can be seen in Fig. 7 with an average relative uncertainty on the cold dust temperatures of 4 per cent and on the warm dust of 10 per cent, $\sim 51$ per cent of pixels are estimated to have cold dust temperatures outside of the standard MAGPHYS priors, with $\sim 32$ per cent of pixels estimate to have warm dust temperatures outside of the standard range. It appears there may be a dust population with $\mathrm{T}<10 \mathrm{~K}$, although this is most likely due to MAGPHYs using a fixed $\beta$ of 2 - Xilouris et al. (2012) find that with a fixed $\beta$ of 1.5 , the minimum dust temperature is $11 \mathrm{~K}$. This could also be due to the sub-mm excess in M33 (Hermelo et al. 2016) causing MAGPHYs to fit colder dust components. However, performing the fits without the $850 \mu \mathrm{m}$ data (where this submillimetre excess is most apparent) produced very little change in the cold dust temperatures. It also appears from the right-hand panel of Fig. 7 that a significant number of pixels are hitting the lower bound of the priors for warm dust temperature. This would indicate that these pixels are well fitted by a single-temperature blackbody fit, but as MAGPHYs enforces two temperatures to be fitted, this effect is unavoidable. A comparison of the reduced $\chi^{2}$ of the MBB and MAGPHYS fits (Fig. 8) finds a median offset of -1.08 , indicating that on average, the MAGPHYS fits tend to be slightly worse.

A comparison of these two dust maps is shown in Fig. 9. The two methods yield very similar results, and a very tight relationship with an RMS scatter of 0.10 . There is a median offset of 0.02 , indicating that the MAGPHYS calculated dust masses tend to be slightly higher. This is not unexpected, as the MBB has been fitted with a variable $\beta$. Many of the fitted $\beta$ values are less than 2 (the value MAGPHYS uses for its cold dust temperature), which will result in the MBB fitting higher dust temperatures, and thus lower dust masses. MAGPHYS takes into account a variety of dust compositions and temperatures, but this more sophisticated modelling does not greatly affect the calculated dust masses. As there is only a slight deviation from the one-to-one relation, this indicates that the MAGPHYS warm dust temperatures often running up against the lower bounds of the parameter space is not having a significant impact on the calculated dust masses.

The total gas mass can be calculated from dust masses using a dust-to-gas ratio (DGR). Sandstrom et al. (2013) find that the DGR is well correlated with metallicity, with a relationship given by

$\log (\mathrm{DGR})=0.55[12+\log (\mathrm{O} / \mathrm{H})]-6.50$.

We combined this with work from Rosolowsky \& Simon (2008), who find the metallicity gradient within M33 to take the form

$12+\log (\mathrm{O} / \mathrm{H})=-0.027 R+8.36$

where $R$ is in $\mathrm{kpc}$. Combining these two, the radial variation in DGR is

$\log (\mathrm{DGR})=-0.015 R-1.902$. 

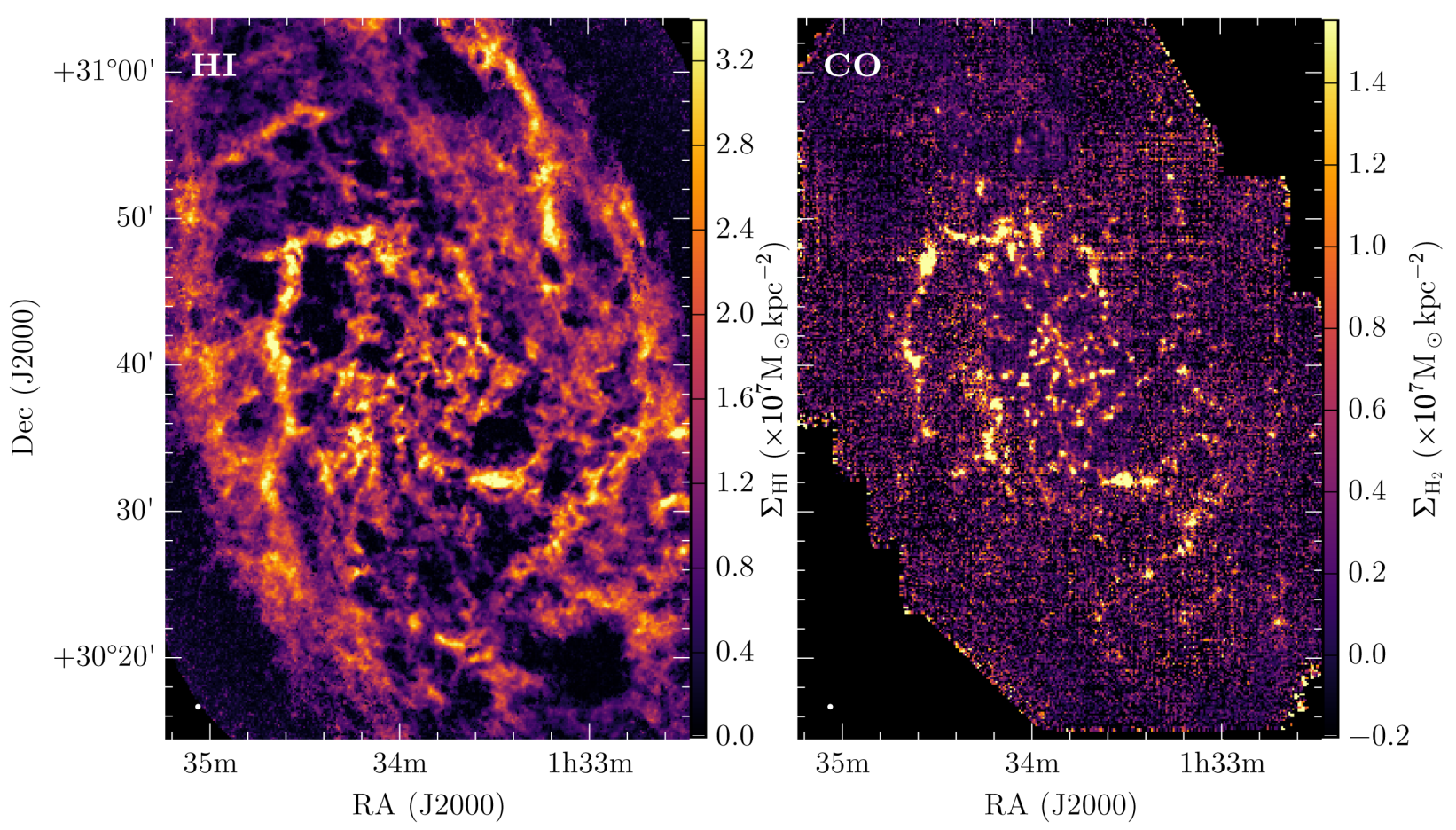

Figure 5. Left: Atomic gas mass surface density calculated from integrated $\mathrm{HI}$ emission (Gratier et al. 2010). Right: Molecular gas mass surface density calculated from integrated $\mathrm{CO}(J=2-1)$ emission (Gratier et al. 2010; Druard et al. 2014). The beam is indicated as a white circle in the lower left.

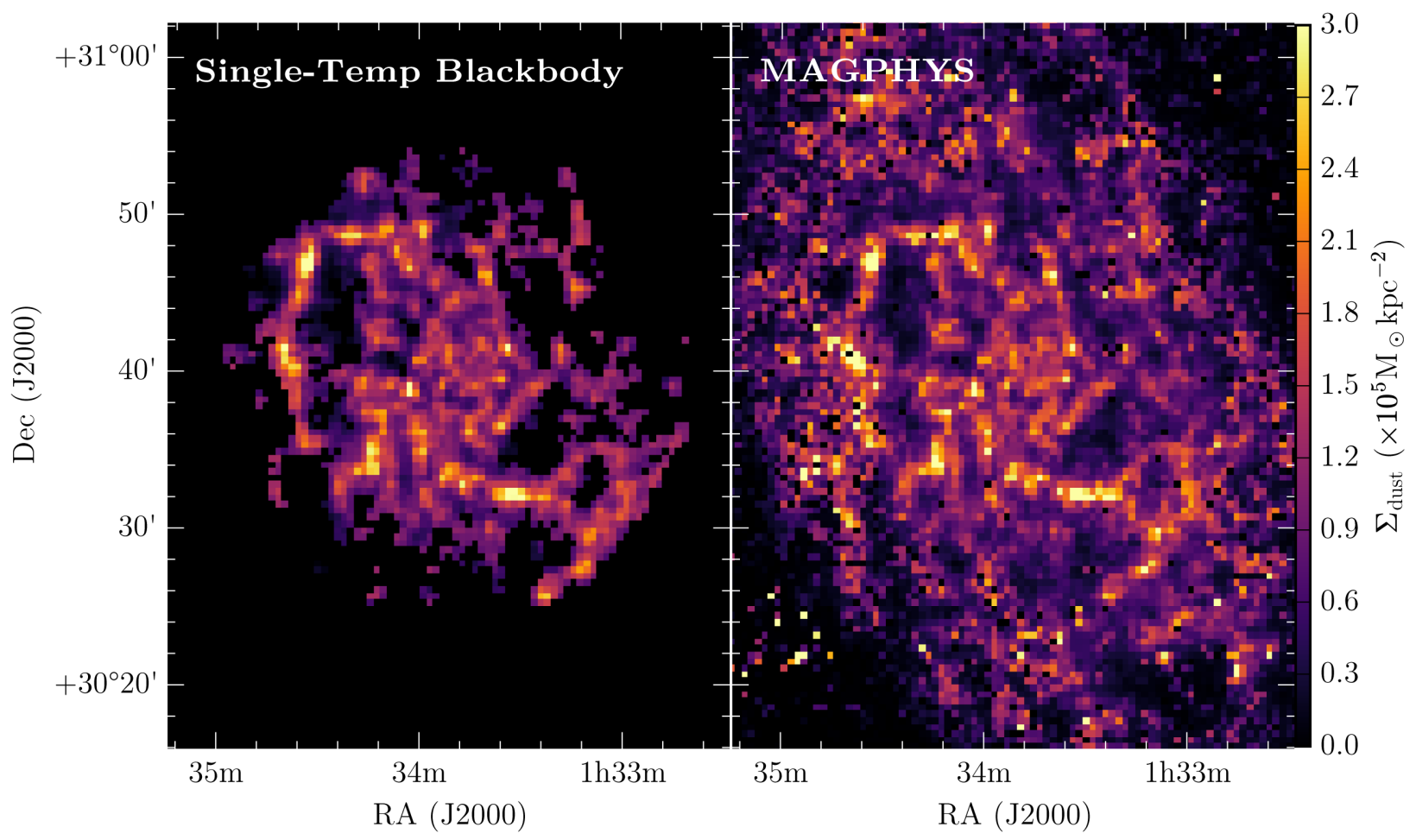

Figure 6. Left: Dust mass surface density from one-temperature greybody fit. Right: Dust mass surface density from MAGPHYs.

We note this gives similar results for the gas-to-dust ratio as the MW ( $\sim 100$, Spitzer 1978), rather than the much higher results of 200 400 found in M33 by Gratier et al. (2017). We find total gas masses of $2.25 \times 10^{8} \mathrm{M}_{\odot}$ (MBB fits) and $9.75 \times 10^{8} \mathrm{M}_{\odot}$ (MAGPHYs).

\subsection{Which gas tracer best correlates with SFR?}

To find out which of these gas maps best traced the SFR, we performed a series of Spearman's rank and Pearson correlation coefficient tests between these gas maps and the MAGPHYS calculated SFR. We also included a total gas map, combining the $\mathrm{HI}$ and $\mathrm{CO}(J=2-1)$ 

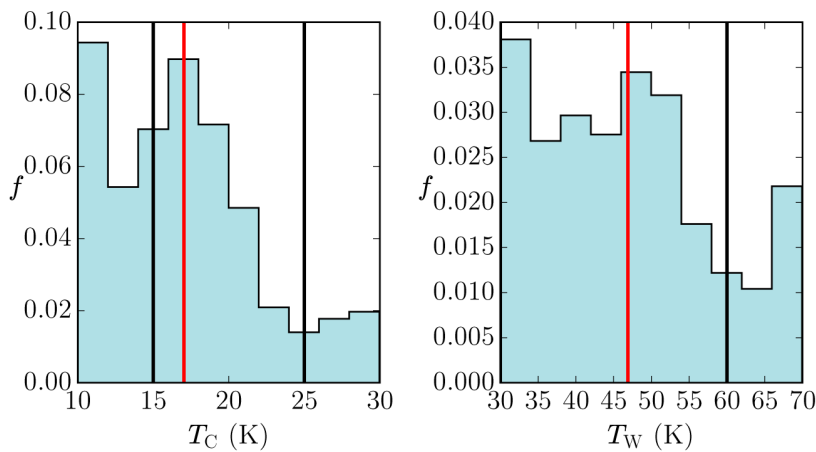

Figure 7. Distribution of cold and warm dust temperatures from MAGPHYS. The red line shows the average value of the sample, the black lines the bounds of the standard MAGPHYS parameter space.

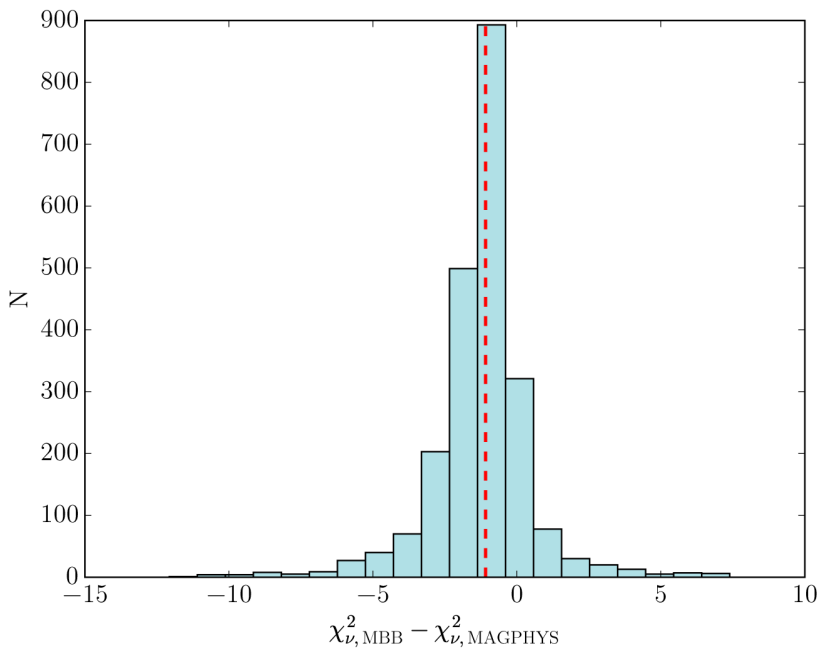

Figure 8. Comparison of reduced $\chi^{2}$ between MBB and MAGPHYS fits for individual pixels. The red dashed line indicates the median of the distribution $(-1.08)$

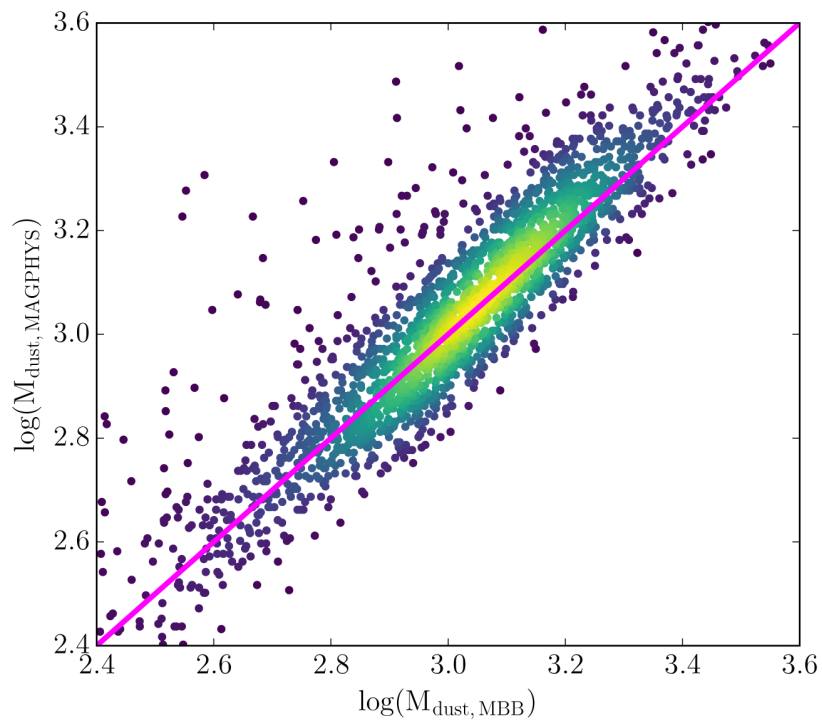

Figure 9. Comparison between the dust masses from MAGPHYS and MBB fitting. The one-to-one relation is shown as a fuchsia line. Blue points indicate a lower point density, yellow a higher point density. data. If the star formation law breaks down at scales of $\sim 100 \mathrm{pc}$, we would expect only a weak correlation here, so we regridded this data to 50 arcsec pixels (corresponding to $200 \mathrm{pc}$ ), to mitigate against this effect.

The correlations between the MAGPHYS SFR and the various gas maps can be seen in Table 3. We find that the correlations between gas and SFR are weaker than those found when comparing integrated galaxies (which typically have $\rho_{\text {sp }} \sim 0.8$ ). This indicates much more scatter on these sub-kpc regions. Of the line-based gas tracers, we find that molecular gas has the strongest correlation this is not surprising, as work by, e.g. Bigiel et al. (2008) has also shown that molecular gas correlates better with SFR than the atomic gas, or the sum of the two.

Of the total gas traced by dust, we find that the MAGPHYS dust fits correlate better than the modified blackbody dust map. This is probably due to MAGPHYS more effectively tracing the total dust continuum, whereas the blackbody is only fitting to the cold dust. We elect to perform our analysis on the molecular gas, the total gas, and the total gas traced by dust from MAGPHYS fitting going forward.

\section{THE STAR FORMATION LAW}

In this section, we investigate the star formation law within M33 at high resolution. We start by comparing the average SFR and gas density to that of other galaxies in previous studies, before performing pixel-by-pixel fits within the galaxy. At high resolution, we also look at the radial variation in the Schmidt index. We have also investigated the effect of the spatial resolution used in the calculation of the KS index, as well as depletion time-scale. Finally, we look at the relationship between SFR and dense gas at high resolution in M33.

\subsection{Global fits}

Fig. 10 shows the mean surface density of SFR and gas for M33 compared with various other galaxies. The mean values here have been calculated from any pixels in the relevant gas map that match up with a pixel from the filtered MAGPHYS SFR map. In the case of the molecular gas, the surface density of SFR is about an order of magnitude higher than expected from this relationship. It is, however, consistent in terms of its molecular gas surface density with work by Ford et al. (2013) on M31, with this higher $\Sigma_{\text {SFR }}$ due to its higher star formation rate and smaller size. The total gas, and gas from dust values lie on the trend. This is unsurprising, as literature values compare SFR surface density with total, rather than molecular gas. Globally, these values are similar to other galaxies, with a somewhat higher star formation efficiency than M31. Overall, we find that the total surface density of gas and SFR is consistent with previous studies - hence, M33 is a typical galaxy in terms of these parameters.

\subsection{Pixel-by-pixel fitting}

We also investigated the star formation law on a pixel-by-pixel basis in M33. For this, we used all pixels in each gas map that matched up with a pixel in the SFR map, performing no S/N cut on the gas data, although by filtering the SFR map, we have effectively performed a S/N cut on the SFR map. This SFR cut biases the data somewhat, although we find that repeating the fits with the unfiltered data produces results consistent within the error bars. With pixel sizes of $100 \mathrm{pc} \times 100 \mathrm{pc}$, we converted the SFR and gas maps into units of surface density. These points can be seen for our three gas tracers in 
Table 3. Correlation coefficients between the various gas maps and the MAGPHYS SFR. All correlations have $p<0.025$.

\begin{tabular}{lccccc}
\hline & $\mathrm{H} \mathrm{I}_{\mathrm{I}}$ & $\mathrm{H}_{2}$ & Total gas & Total gas from single-temp. blackbody & Total gas from MAGPHYs \\
\hline$\rho_{\text {sp }}$ & 0.35 & 0.57 & 0.42 & 0.44 & 0.51 \\
$\rho_{\text {pears }}$ & 0.29 & 0.55 & 0.37 & 0.41 & 0.51 \\
\hline
\end{tabular}

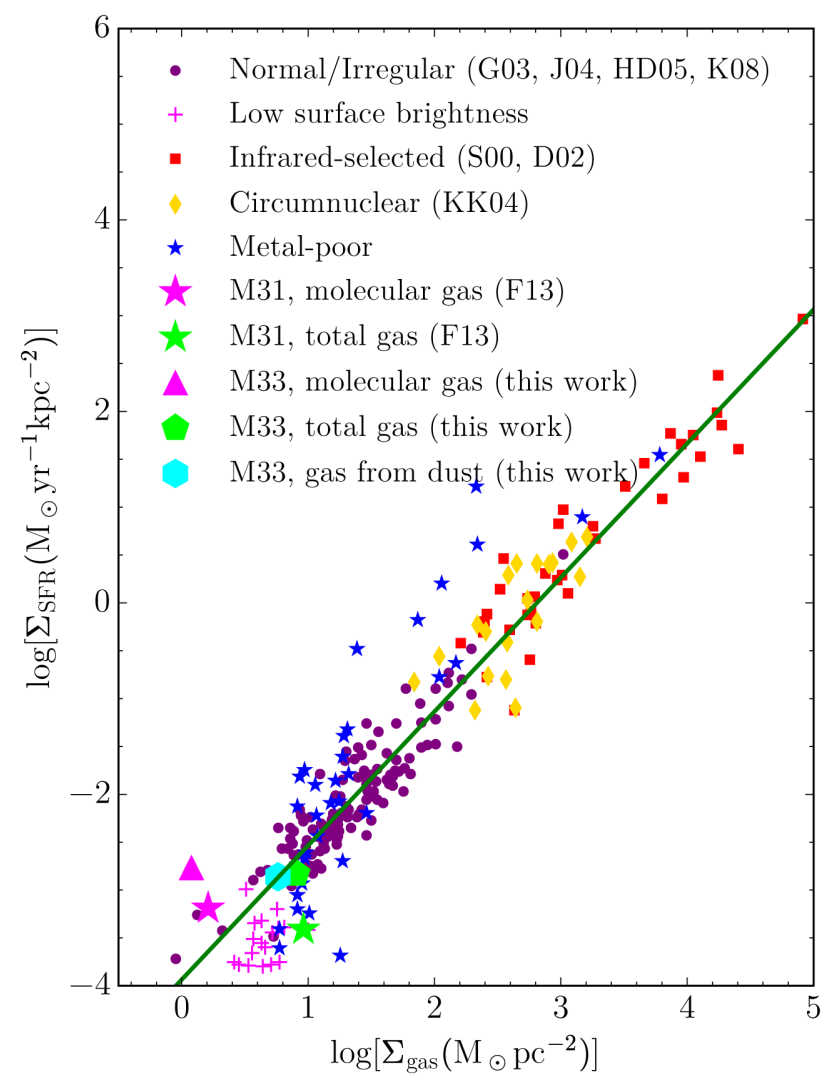

Figure 10. Surface density of SFR against surface density of gas for a variety of galaxies. Purple circles indicate the normal and irregular galaxies of Gavazzi et al. (2003), James et al. (2004), Hameed \& Devereux (2005), and Kennicutt et al. (2008). Fuchsia pluses show the subset of these galaxies classified as low-surface brightness. The red squares are infrared-selected starburst galaxies of Scoville et al. (2000) and Dopita et al. (2002), with circumnuclear starburst galaxies from Kormendy \& Kennicutt (2004) shown as yellow diamonds. The subset of all of these galaxies with metal abundances, $Z<0.3 Z_{\odot}$ are shown as blue stars. The values for molecular and total gas for M31 (Ford et al. 2013) are shown as purple and light green stars, respectively. The purple triangle, light green pentagon and light blue hexagon show the values calculated in this work from the molecular gas, total gas, and gas from dust, respectively. Also shown is a green line with slope, $N=1.4$.

the top row of Fig. 11. At spatial scales of 100 pc, although we see significant scatter in the points, we find correlations between the gas and SFR surface densities $\left(\rho_{\mathrm{sp}}=[0.54,0.42,0.37]\right.$ for molecular gas, total gas and gas from dust, respectively). This is in stark contrast to, e.g. Onodera et al. (2010); Schruba et al. (2010), who find very little correlation between these quantities at comparable spatial scales. We attribute this to the methods these works have employed to calculate the SFR in these sub-kpc regions. These works calculated SFR using methods that assume constant star formation over the last $100 \mathrm{Myr}$, which the MAGPHYs fits and work by Relaño \& Kennicutt (2009) show is likely inappropriate over these sub-kpc regions. This can affect the calculated SFR by an order of magnitude (Fig. 4). We find that using our other SFR measures results in much more scatter at the highest resolutions, similar to that shown in Onodera et al. (2010).

Another difference in our method is the choice of molecular gas tracer. Whilst Onodera et al. (2010) and Schruba et al. (2010) opt to use the $J=1-0$ line to trace the molecular gas, we use the $J=2-1$ line. Higher rotational transitions of CO trace warmer, denser, molecular gas, which may be more closely associated with star formation. Work has shown that higher rotational transitions are more strongly correlated with SFR (e.g. Komugi et al. 2007; Bayet et al. 2009), and so this stronger correlation would be expected.

To calculate a Schmidt index, we use orthogonal distance regression (ODR), to account for errors in both $\Sigma_{\mathrm{SFR}}$ and $\Sigma_{\text {gas }}$. The error in $\Sigma_{\text {SFR }}$ comes from the MAGPHYS modelling uncertainties, and the errors in gas surface density are derived from the RMS noise of the particular map (ignoring any calibration error, as this will simply cause an offset to all points and not affect the slope). In the case of the MAGPHYS dust map, we use the relative error calculated from the percentiles of the PDF. We perform this fit in linear space, as these error bars will be asymmetrical in log space. We fit this line to the entire data set, and these fits can be seen in Fig. 11. With the large range of S/N values of our $\Sigma_{\mathrm{SFR}}$ and $\Sigma_{\text {gas }}$, particularly the large number of low-S/N points, it is necessary to account for uncertainties in both variables when performing the model fitting. The effects of assuming a constant fractional uncertainty for all the data points rather than our measured uncertainty estimates is discussed in Appendix.

At the highest resolutions, we find three very different indices to the classic $N=1.4$. Even in the case of molecular gas where we see the strongest correlations, the Schmidt index is higher than we would expect from Kennicutt (1998a). To our knowledge, these values are higher than others previously reported. However, with strong correlations remaining between $\Sigma_{\text {SFR }}$ and the molecular gas, we argue that the star formation law holds at these small scales. It also appears that the dust traces the total, rather than molecular gas.

\subsection{A search for a radial variation in $N$}

Works such as Leroy et al. (2008) and Ford et al. (2013) have shown that a radial variation can be seen in the Schmidt index in some galaxies. To investigate this at high resolution in M33, we have taken the pixels inside five annuli of constant galactocentric radius from the centre of M33 to $1.2 R_{25}$. The results of this can be seen in Fig. 12. We see little radial variation in $N$ for all three tracers of gas, indicating that the star formation efficiency is reasonably constant across the disk of M33. These results for all three gas tracers appear very different to those of Leroy et al. (2008) for spiral galaxies in general and Ford et al. (2013) for M31 in particular. We find that the calculated value of $N$ is reasonably consistent with each tracer of gas for these radial bins, with a peak in the outer spiral arms for the total gas, and the gas from dust. 


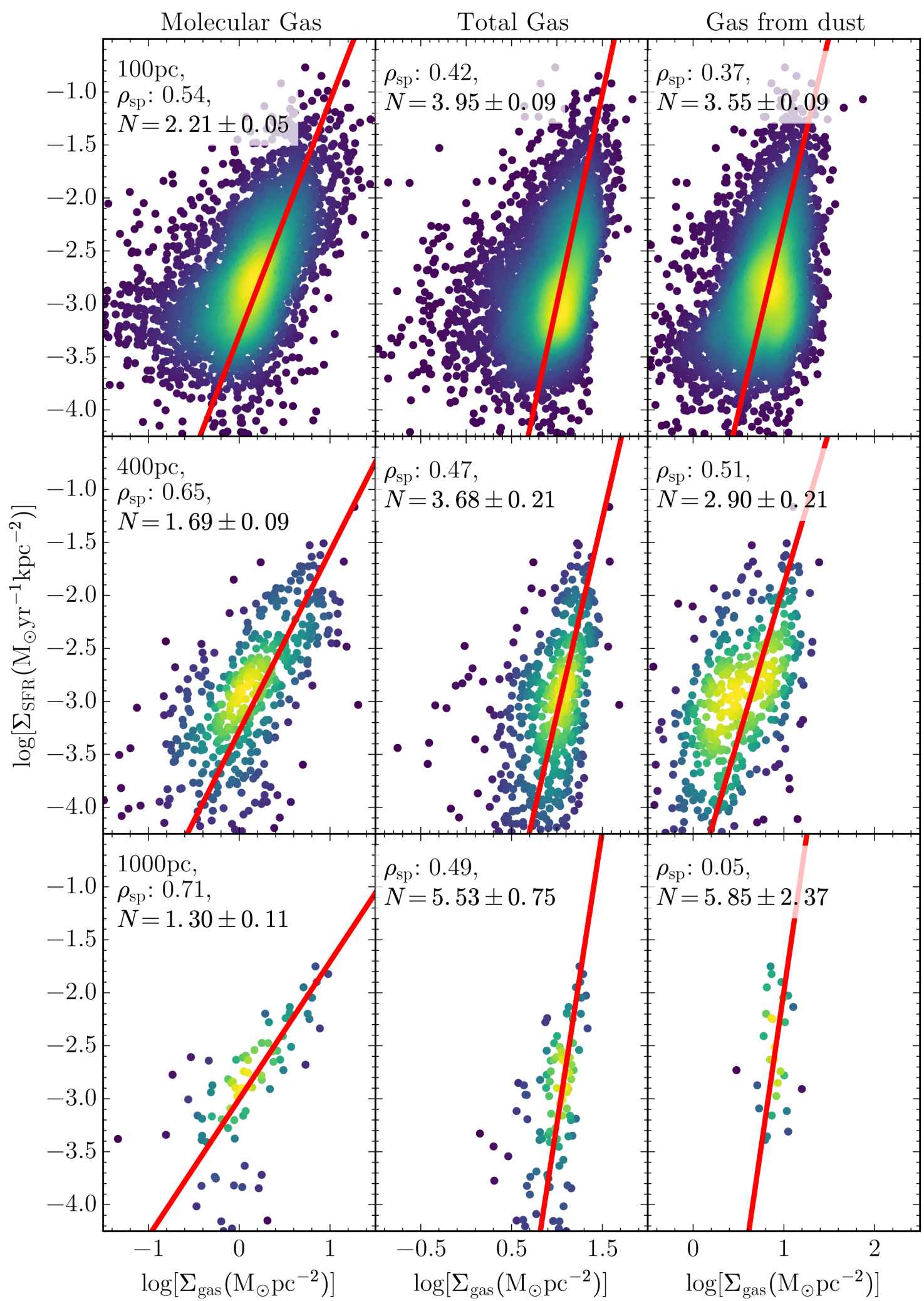

Figure 11. Left column: SFR surface density against molecular gas surface density. Middle column: SFR surface density against total gas surface density. Right column: SFR surface density against gas surface density, as traced by dust. The points are coloured by point density, from blue (low density) to yellow (high density). The red line indicates the best fit in each case. 

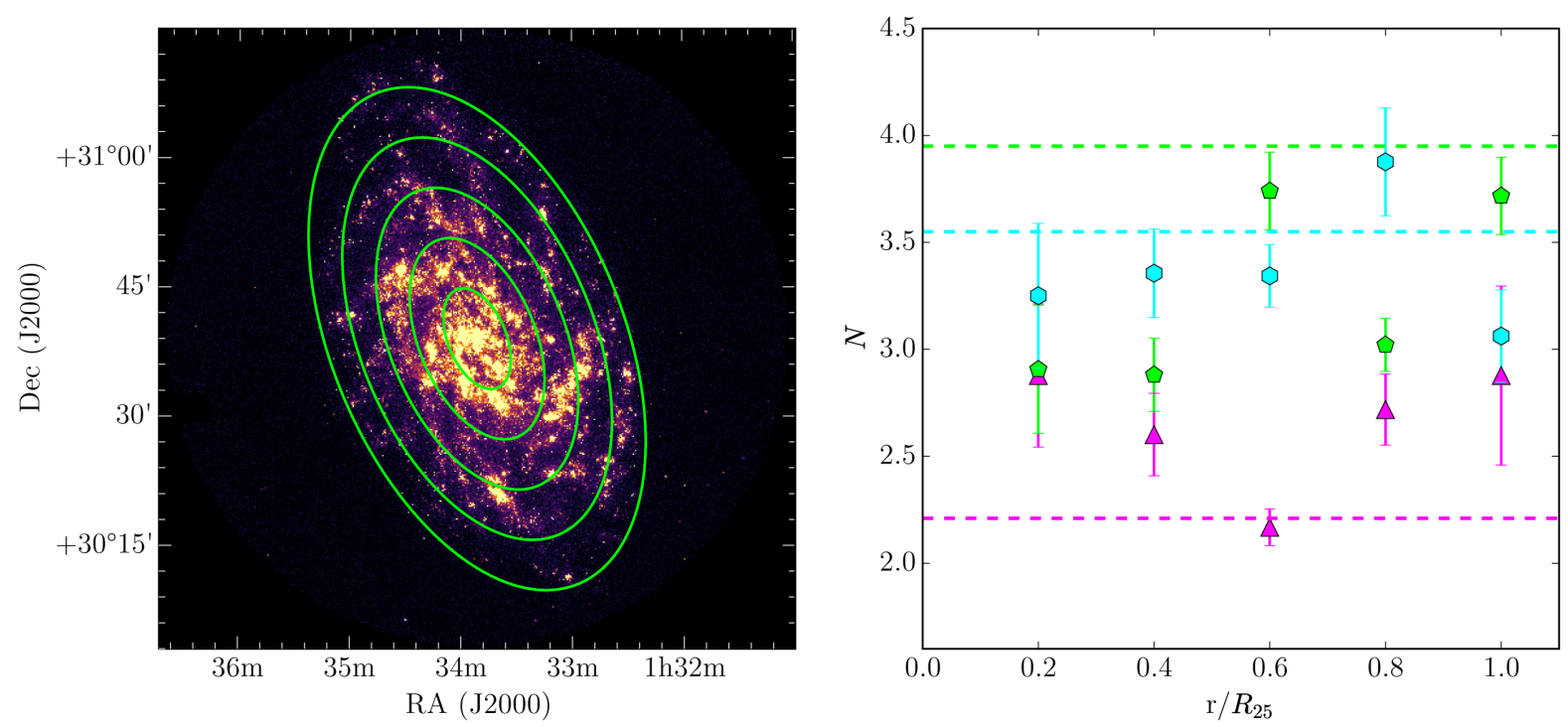

Figure 12. Schmidt index with galactocentric radius within M33. Purple triangles indicate values calculated using the molecular gas, light green pentagons from the total gas, and light blue hexagons from the gas traced by dust. Also shown are dashed lines indicating the average value of $N$ fitted to all pixels.

\subsection{Variation with pixel scale}

Although we find correlation at scales of $100 \mathrm{pc}$, these are not as strong as those found by, e.g. Schruba et al. (2010) in M33 ( $\rho_{\text {sp }} \sim 0.8$ at $1200 \mathrm{pc}$ resolution). We expect this correlation to increase, and scatter between points to decrease with increasing pixel scale - we average over GMCs in various evolutionary states at larger spatial scales, and it is this that is believed to drive the KS relation (Onodera et al. 2010). In order to test this increase in correlation, we regridded our data to a number of pixel scales ( $50-500$ arcsec, 200 pc to $2 \mathrm{kpc}$ ). For the gas maps, we performed this using MONTAGE's MPROJECT routine, and for any quantities derived from MAGPHYs we ran the fits on the regridded data. We then performed a fit to each pixel scale, using the same method as detailed in Section 5.2. A selection of these fits can be seen in the lower panels of Fig. 11, and the calculated values of $N$, along with Spearman's rank correlations can be seen in Table 4 . We also indicate the less statistically significant (i.e. $p>0.025)$ correlations with an asterisk $(*)$.

Between pixel scales and gas tracers, we see variation in the Schmidt index. With molecular gas, this decreases with increasing pixel scale before becoming approximately linear at a scale of $\sim 2 \mathrm{kpc}$. This value is consistent with the index found by Bigiel et al. (2008) when considering molecular gas, and indicates that at kpc resolutions, these GMC populations appear much more uniform, and we are simply counting the numbers of them. We also find that, in general, $N$ increases with increasing pixel scale for the total gas. There is, however, significant scatter in our calculated $N$ with pixel scale with gas from dust, and at large pixel scales the correlation between this tracer of gas and SFR is no longer statistically significant. For our more conventional gas tracers, we find an increasing correlation between the surface density of gas and SFR with increasing pixel scale, with decreasing scatter from the relationship. This would indicate that the star formation law we see at integrated galaxy scales is driven by an average of GMCs at various evolutionary states in a galaxy.

We also investigate the scale dependence on the gas depletion time-scale - this can be seen in Fig. 13. We calculate the depletion time-scale in the same manner as Schruba et al. (2010), where $\tau_{\text {dep }}$ $=\Sigma_{\text {gas }} / \Sigma_{\mathrm{SFR}}$. We quote the $1 \sigma$ errors based on the percentiles of the depletion time-scale distribution. Unlike Schruba et al. (2010), we find no significant variation in the gas depletion time-scale with resolution, for any of our three gas tracers. This would appear to be due to their targeted selection of only the brightest regions of $\mathrm{CO}$ and $\mathrm{H} \alpha$ - when taking into account the entire ensemble of regions within M33, these resolution effects are no longer significant.

It is also important to note that these results cover a much smaller dynamic range than Fig. 10, and so the correlations would naturally be weaker. We took a subset of the data in Fig. 10 over the $\sim 2$ orders of magnitude that our data covers. We found that the average Spearman correlation coefficient is $\sim 0.84$ for these data, still stronger than we find for M33 at any spatial scale. To recover the correlation we see comparing galaxy to galaxy, it seems necessary to take into account the entire ensemble of GMCs within that particular galaxy.

We find that these data also do not reproduce the correlations seen by Schruba et al. (2010), who find a correlation coefficient of $\sim 0.8$ at scales of $\sim 1 \mathrm{kpc}$. However, their work used targeted apertures on $\mathrm{CO}$ and $\mathrm{H} \alpha$ peaks, biasing their results towards areas of high star formation and $\mathrm{S} / \mathrm{N}$, where this star formation law holds more strongly. When we place apertures centered on peaks of gas or SFR, rather than pixel-by-pixel comparisons, we find a stronger correlation ( $\rho_{\mathrm{sp}}=0.82$ for apertures of $1200 \mathrm{pc}$ diameter), comparable with that of Schruba et al. (2010). Our work blindly includes all areas within a galaxy, so we avoid the very high $\mathrm{S} / \mathrm{N}$ requirements of Schruba et al. (2010). Thus, this is the cause of these slightly weaker correlations.

\subsection{SFR and dense gas}

We have investigated the relationship between dense gas and SFR, to see if this relationships show a similar scale dependence to the star formation law. We have used $\operatorname{HCN}(J=1-0)$ emission to trace the dense molecular gas. Most of these dense gas pointings come from Buchbender et al. (2013), with some complementary measurements from Rosolowsky, Pineda \& Gao (2011) and Braine et al. (2017). All of these pointings were from the IRAM $30 \mathrm{~m}$ telescope, with a beam size of 28 arcsec, comparable to our pixel size. To convert the $\mathrm{HCN}$ luminosity to a mass of dense $\mathrm{H}_{2}$, we used a conversion factor, $\alpha_{\mathrm{HCN}}=10$ (Gao \& Solomon 2004a). The results of this 
Table 4. Schmidt index for the molecular gas, total gas, and gas from dust, for a variety of pixel scales. Asterisks $(*)$ indicate correlations with $p>0.025$.

\begin{tabular}{lcccccc}
\hline & \multicolumn{2}{c}{ Molecular gas } & & Total gas & \multicolumn{2}{c}{ Gas from dust } \\
Scale $(\mathrm{pc})$ & $N$ & $\rho_{\text {sp }}$ & $N$ & $\rho_{\text {sp }}$ & $\rho_{\text {sp }}$ \\
\hline 100 & $2.21 \pm 0.05$ & 0.54 & $3.95 \pm 0.09$ & 0.42 & $3.55 \pm 0.09$ \\
200 & $1.90 \pm 0.06$ & 0.57 & $1.93 \pm 0.12$ & 0.42 & $3.13 \pm 0.09$ \\
400 & $1.69 \pm 0.09$ & 0.65 & $3.68 \pm 0.21$ & 0.47 & $2.90 \pm 0.21$ & 0.37 \\
600 & $1.51 \pm 0.09$ & 0.70 & $3.90 \pm 0.29$ & 0.52 & 0.51 \\
1000 & $1.30 \pm 0.11$ & 0.71 & $5.53 \pm 0.75$ & 0.49 & $5.85 \pm 2.37$ \\
2000 & $1.07 \pm 0.16$ & 0.87 & $5.20 \pm 1.33$ & 0.50 & $0.07 \pm 0.36$ \\
\hline
\end{tabular}

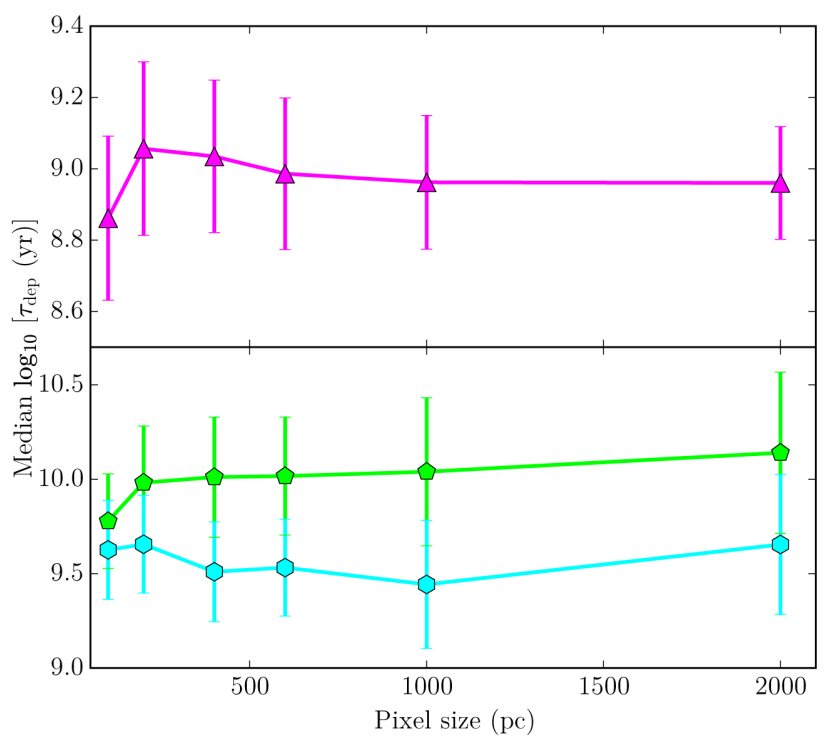

Figure 13. Depletion time-scales for the three gas tracers with pixel size. Purple triangles indicate the molecular gas, green pentagons the total gas, and light blue hexagons the gas traced by dust.

can be seen in Fig. 14 - a strong correlation is seen between the dense gas mass and SFR $\left(\rho_{\mathrm{sp}}=0.68, p=0.04\right)$. Additionally, these points lie approximately on the extrapolation of the linear fit seen for integrated galaxies, indicating that this dense gas relation holds down to these $100 \mathrm{pc}$ scales. Possibly the reason these points are somewhat below the line is due to the low metallicity of M33, since due to their sensitivity to photodissociation, dense gas tracers are very strongly dependent on metallicity (Rosolowsky et al. 2011). Nevertheless, these results indicate that the dense molecular gas correlates more strongly than the regular molecular gas with SFR at these sub-kpc scales.

\section{DISCUSSION AND CONCLUSIONS}

In this work, we have presented a high-resolution study of the star formation law in M33 on spatial scales of $\sim 100$ pc. By assembling GALEX UV, SDSS, WISE, Spitzer, Herschel, Planck, and new SCUBA-2 observations we have measured the SFR using the panchromatic SED fitting tool MAGPHYS. We have compared this SFR with that calculated both by the TIR luminosity and FUV $+24 \mu \mathrm{m}$ data. We find that much of the starlight in M33 is unattenuated by dust, leading to an underestimate of the total SFR from the TIR luminosity. MAGPHYS models many of the HII regions of M33 as starburst-like, and thus produces pixels with much higher SFR than even the FUV $+24 \mu \mathrm{m}$ calculated SFR. Since MAGPHYS uses all available data and allows variations in SFR to much shorter time-

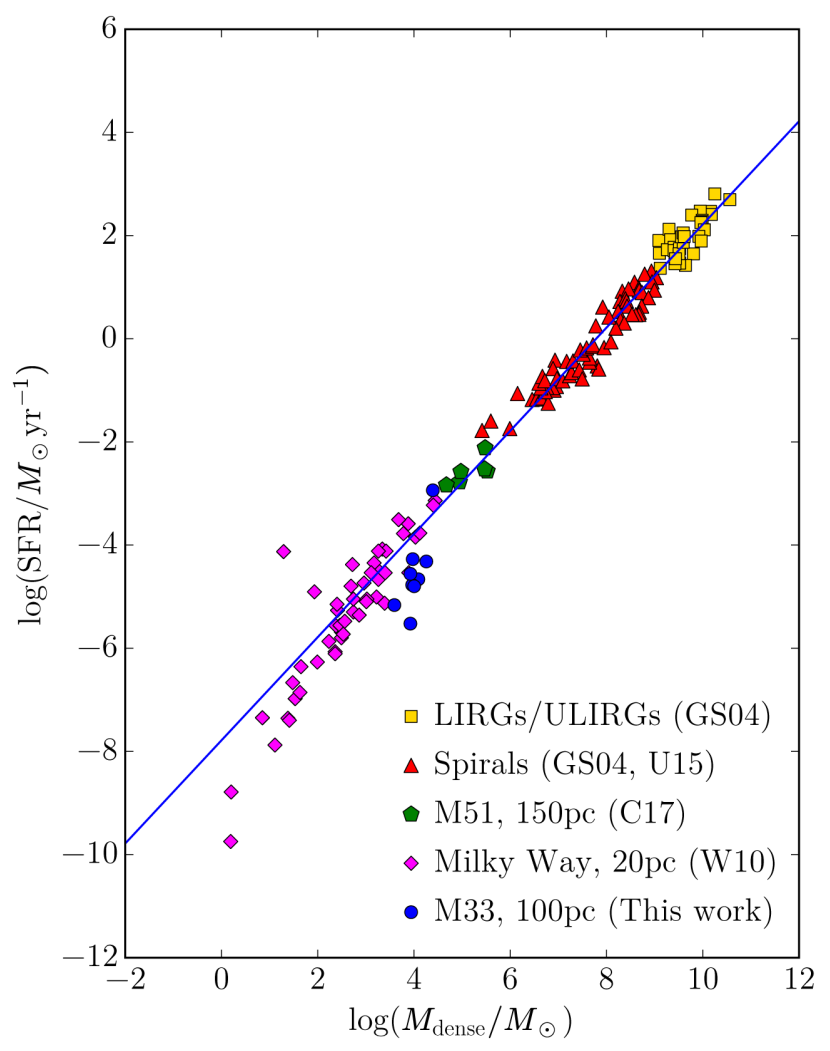

Figure 14. Relationship between SFR and dense gas mass across a range of environments and scales. Yellow squares indicate values for the LIRGs and ULIRGs of Gao \& Solomon (2004b), red triangles spiral galaxies from the surveys of Gao \& Solomon (2004b) and Usero et al. (2015). Green pentagons are sub-kpc measurements taken in M51 by Chen et al. (2017), purple diamonds are from the MW pointings of Wu et al. (2010). Blue circles indicate values for this work - SFR values have been calculated using MAGPHYS, and dense gas pointings come from a variety of sources, described in the main text. In every case, the dense gas mass has been calculated using $\mathrm{HCN}(J=1-0)$, and for pointings that are resolved, the approximate spatial scale is given in the legend. The blue line indicates a linear fit to the data.

scales than the TIR and FUV+24 $\mu$ m prescriptions, we have used this in our analysis.

We have combined the SFR calculated from these SED fits with gas maps created from $\mathrm{HI}$ and $\mathrm{CO}(J=2-1)$ data. We have also constructed dust mass maps of M33 using both a MBB fitting code and MAGPHYS, and compared the two. We find that these two maps agree very closely, with the MAGPHYS masses tending to be slightly higher - not unexpected with a variable $\beta$ and a single temperature in the case of the MBB fitting. It is important to note that when fitting a two-temperature MBB to M33, Tabatabaei et al. (2014) find that a fixed $\beta$ of 1.5 for the cold dust component was a better 
fit than the 2 the MAGPHYS uses, but the effect of this is minor. Using the DGR calculated from the metallicity gradient, we turn these dust maps into total gas mass maps. We use maps of the molecular gas, total gas $(\mathrm{CO}+\mathrm{HI})$ and total gas from dust to probe the star formation law at scales of $100 \mathrm{pc}$.

We find that M33 is not an unusual galaxy in terms of its overall gas and SFR surface density, and whilst correlations remain down to scales of $100 \mathrm{pc}$, the measured Schmidt index shows a strong scale dependence. This indicates that the GMCs within M33 are at a variety of evolutionary states, and so the star formation law is very different at GMC, rather than galaxy scales. We also find that at these scales, molecular gas better traces SFR. The gas depletion time-scale, however, shows no such scale dependence. We find that $N$ is reasonably invariant with galactocentric radius, with a peak in $N$ for total gas and gas from dust in the outer spiral arms. If we consider the dense gas mass of a galaxy, a tight, linear relationship is found, perhaps indicating that dense molecular gas is the fundamental building block of star formation.

Using a wide range of high-resolution data, and leveraging the close proximity of M33, we have been able to probe the light at GMC scales in this galaxy across some 4 orders of magnitude in wavelength. From this broad range of coverage, a large range of galaxy parameters can be calculated, and various laws probed down to the small-scale. It would appear that, at the scales of GMCs, the star formation law does hold, although the Schmidt index is very different at these scales. We also find a quasi-universal star formation law with dense molecular gas.

\section{ACKNOWLEDGEMENTS}

The authors would like to thank the reviewer for helpful comments, Stephen Eales and Jenifer Millard for valuable discussions, Sébastien Viaene for providing the extended MAGPHYS IR libraries, and Elisabete da Cunha for MAGPHYs assistance. TGW would also like to personally thank Ken Fields.

MWLS acknowledges support from the European Research Council (ERC) Forward Progress 7 (FP7) Herschel Extragalactic Legacy Project (HELP). The James Clerk Maxwell Telescope is operated by the East Asian Observatory on behalf of The National Astronomical Observatory of Japan, Academia Sinica Institute of Astronomy and Astrophysics, the Korea Astronomy and Space Science Institute, the National Astronomical Observatories of China and the Chinese Academy of Sciences (grant no. XDB09000000), with additional funding support from the Science and Technology Facilities Council of the United Kingdom and participating universities in the United Kingdom and Canada.

This research has made use of the NASA/IPAC Infrared Science Archive, which is operated by the Jet Propulsion Laboratory, California Institute of Technology, under contract with the National Aeronautics and Space Administration, as well as the NASA/IPAC Extragalactic Database (NED) which is operated by the Jet Propulsion Laboratory, California Institute of Technology, under contract with the National Aeronautics and Space Administration.

This research made use of Montage, which is funded by the National Science Foundation under Grant Number ACI-1440620, and was previously funded by the National Aeronautics and Space Administration's Earth Science Technology Office, Computation Technologies Project, under Cooperative Agreement Number NCC5-626 between NASA and the California Institute of Technology.

This research made use of Astropy, a community-developed core Python package for Astronomy (Astropy Collaboration, 2013).

\section{REFERENCES}

Alam S. et al., 2015, ApJS, 219, 12

André P. et al., 2010, A\&A, 518, L102

Aniano G., Draine B. T., Gordon K. D., Sandstrom K., 2011, PASP, 123, 1218

Bayet E., Gerin M., Phillips T. G., Contursi A., 2009, MNRAS, 399, 264

Bertin E., Arnouts S., 1996, A\&AS, 117, 393

Bigiel F., Leroy A., Walter F., Brinks E., de Blok W. J. G., Madore B., Thornley M. D., 2008, AJ, 136, 2846

Bigiel F., Leroy A. K., Blitz L., Bolatto A. D., da Cunha E., Rosolowsky E., Sandstrom K., Usero A., 2015, ApJ, 815, 103

Boquien M. et al., 2015, A\&A, 578, A8

Boselli A., Boissier S., Cortese L., Buat V., Hughes T. M., Gavazzi G., 2009, ApJ, 706, 1527

Braine J. et al., 2010, A\&A, 518, L69

Braine J., Shimajiri Y., André P., Bontemps S., Gao Y., Chen H., Kramer C., 2017, A\&A, 597, A44

Bruzual G., Charlot S., 2003, MNRAS, 344, 1000

Buchbender C. et al., 2013, A\&A, 549, A17

Calzetti D. et al., 2005, ApJ, 633, 871

Calzetti D. et al., 2007, ApJ, 666, 870

Chabrier G., 2003, PASP, 115, 763

Chapin E. L., Berry D. S., Gibb A. G., Jenness T., Scott D., Tilanus R. P. J., Economou F., Holland W. S., 2013, MNRAS, 430, 2545

Charlot S., Fall S. M., 2000, ApJ, 539, 718

Chen H., Braine J., Gao Y., Koda J., Gu Q., 2017, ApJ, 836, 101

Clark C. J. R., Schofield S. P., Gomez H. L., Davies J. I., 2016, MNRAS, 459, 1646

Corbelli E., Thilker D., Zibetti S., Giovanardi C., Salucci P., 2014, A\&A, 572, A23

Cortese L., Boselli A., Franzetti P., Decarli R., Gavazzi G., Boissier S., Buat V., 2008, MNRAS, 386, 1157

da Cunha E., Charlot S., Elbaz D., 2008, MNRAS, 388, 1595

Dale D. A. et al., 2009, ApJ, 703, 517

Dempsey J. T. et al., 2013, MNRAS, 430, 2534

Dopita M. A., Pereira M., Kewley L. J., Capaccioli M., 2002, ApJS, 143, 47

Druard C. et al., 2014, A\&A, 567, A118

Dunne L., Eales S., Edmunds M., Ivison R., Alexander P., Clements D. L., 2000, MNRAS, 315, 115

Eales S. et al., 2012, ApJ, 761, 168

Eckart A., Downes D., Genzel R., Harris A. I., Jaffe D. T., Wild W., 1990, ApJ, 348, 434

Elmegreen B. G., 1994, ApJ, 425, L73

Engelbracht C. W. et al., 2007, PASP, 119, 994

Fazio G. G. et al., 2004, ApJS, 154, 10

Ford G. P. et al., 2013, ApJ, 769, 55

Gao Y., Solomon P. M., 2004a, ApJS, 152, 63

Gao Y., Solomon P. M., 2004b, ApJ, 606, 271

Gao Y., Zhang Z., Greve T., MALATANG Team, 2017, AAS Meeting Abstracts, p. 324.01

García-Burillo S., Usero A., Alonso-Herrero A., Graciá-Carpio J., PereiraSantaella M., Colina L., Planesas P., Arribas S., 2012, A\&A, 539, A8

Gardan E., Braine J., Schuster K. F., Brouillet N., Sievers A., 2007, A\&A, 473,91

Gavazzi G., Boselli A., Donati A., Franzetti P., Scodeggio M., 2003, A\&A, 400, 451

Gordon K. D. et al., 2007, PASP, 119, 1019

Gratier P. et al., 2010, A\&A, 522, A3

Gratier P. et al., 2017, A\&A, 600, A27

Griffin M. J. et al., 2010, A\&A, 518, L3

Hameed S., Devereux N., 2005, AJ, 129, 2597

Hao C.-N., Kennicutt R. C., Johnson B. D., Calzetti D., Dale D. A., Moustakas J., 2011, ApJ, 741, 124

Hartwick F. D. A., 1971, ApJ, 163, 431

Hermelo I. et al., 2016, A\&A, 590, A56

Heyer M. H., Corbelli E., Schneider S. E., Young J. S., 2004, ApJ, 602, 723

Hildebrand R. H., 1983, QJRAS, 24, 267 
Hirashita H., Inoue A. K., Kamaya H., Shibai H., 2001, A\&A, 366, 83 Ho L. C., Filippenko A. V., Sargent W. L. W., 1997, ApJ, 487, 579

Holland W. S. et al., 2013, MNRAS, 430, 2513

James P. A. et al., 2004, A\&A, 414, 23

Jarrett T. H. et al., 2011, ApJ, 735, 112

Kennicutt R. C., Jr. et al., 2009, ApJ, 703, 1672

Kennicutt R. C., Jr. 1998a, ARA\&A, 36, 189

Kennicutt R. C., Jr. 1998b, ApJ, 498, 541

Kennicutt R. C., Evans N. J., 2012, ARA\&A, 50, 531

Kennicutt R. C. Jr., Lee J. C., Funes J. G. J. S., Sakai S., Akiyama S., 2008, ApJS, 178, 247

Kewley L. J., Geller M. J., Jansen R. A., Dopita M. A., 2002, AJ, 124, 3135

Khoperskov S. A., Vasiliev E. O., 2017, MNRAS, 468, 920

Komugi S., Kohno K., Tosaki T., Nakanishi H., Onodera S., Egusa F., Sofue Y., 2007, PASJ, 59, 55

Kormendy J., Kennicutt R. C., Jr. 2004, ARA\&A, 42, 603

Kramer C. et al., 2010, A\&A, 518, L67

Kroupa P., 2001, MNRAS, 322, 231

Krumholz M. R., McKee C. F., 2005, ApJ, 630, 250

Krumholz M. R., Thompson T. A., 2007, ApJ, 669, 289

Lada C. J., Lombardi M., Alves J. F., 2010, ApJ, 724, 687

Lada C. J., Forbrich J., Lombardi M., Alves J. F., 2012, ApJ, 745, 190

Lee J. C. et al., 2011, ApJS, 192, 6

Leitherer C. et al., 1999, ApJS, 123, 3

Leroy A. K., Walter F., Brinks E., Bigiel F., de Blok W. J. G., Madore B., Thornley M. D., 2008, AJ, 136, 2782

Long K. S., Charles P. A., Dubus G., 2002, ApJ, 569, 204

Madore B. F., Freedman W. L., 1991, PASP, 103, 933

Magdis G. E. et al., 2012, ApJ, 760, 6

Mainzer A. et al., 2011, ApJ, 731, 53

Martin D. C. et al., 2005, ApJ, 619, L1

McConnachie A. W., Ferguson A. M. N., Irwin M. J., Dubinski J., Widrow L. M., Dotter A., Ibata R., Lewis G. F., 2010, ApJ, 723, 1038

Morrissey P. et al., 2007, ApJS, 173, 682

Murgia M., Crapsi A., Moscadelli L., Gregorini L., 2002, A\&A, 385, 412

Murphy E. J. et al., 2011, ApJ, 737, 67

Narayanan D., Krumholz M. R., Ostriker E. C., Hernquist L., 2012, MNRAS, 421,3127

Onodera S. et al., 2010, ApJ, 722, L127

Padmanabhan N. et al., 2008, ApJ, 674, 1217

Paturel G., Theureau G., Bottinelli L., Gouguenheim L., Coudreau-Durand N., Hallet N., Petit C., 2003, A\&A, 412, 57

Pilbratt G. L. et al., 2010, A\&A, 518, L1

Planck Collaboration XVI, 2014, A\&A, 571, A16

Poglitsch A. et al., 2010, A\&A, 518, L2

Putman M. E. et al., 2009, ApJ, 703, 1486

Regan M. W., Vogel S. N., 1994, ApJ, 434, 536

Relaño M., Kennicutt R. C., Jr. 2009, ApJ, 699, 1125

Rieke G. H. et al., 2004, ApJS, 154, 25

Rohlfs K., Wilson T., 1996, Tools of Radio Astronomy. Astronomy and Astrophysics Library, Springer

Rosolowsky E., Simon J. D., 2008, ApJ, 675, 1213

Rosolowsky E., Keto E., Matsushita S., Willner S. P., 2007, ApJ, 661, 830

Rosolowsky E., Pineda J. E., Gao Y., 2011, MNRAS, 415, 1977

Sandstrom K. M. et al., 2013, ApJ, 777, 5

Sanduleak N., 1969, AJ, 74, 47

Schlafly E. F., Finkbeiner D. P., 2011, ApJ, 737, 103

Schmidt M., 1959, ApJ, 129, 243

Schruba A., Leroy A. K., Walter F., Sandstrom K., Rosolowsky E., 2010, ApJ, 722, 1699

Schuster K.-F. et al., 2004, A\&A, 423, 1171

Scoville N. Z. et al., 2000, AJ, 119, 991

Smith M. W. L. et al., 2010, A\&A, 518, L51

Smith M. W. L. et al., 2012, ApJ, 756, 40

Solomon P. M., Rivolo A. R., Barrett J., Yahil A., 1987, ApJ, 319, 730

Spitzer L., 1978, Physical processes in the interstellar medium. Wiley, New York, p.333

Strong A. W., Mattox J. R., 1996, A\&A, 308, L21
Tabatabaei F. S. et al., 2014, A\&A, 561, A95

Thilker D. A. et al., 2005, ApJ, 619, L67

Usero A. et al., 2015, AJ, 150, 115

Verley S., Hunt L. K., Corbelli E., Giovanardi C., 2007, A\&A, 476, 1161

Verley S., Corbelli E., Giovanardi C., Hunt L. K., 2009, A\&A, 493, 453

Verley S., Corbelli E., Giovanardi C., Hunt L. K., 2010, A\&A, 510, A64

Viaene S. et al., 2014, A\&A, 567, A71

Wolfe S. A., Pisano D. J., Lockman F. J., McGaugh S. S., Shaya E. J., 2013, Nature, 497, 224

Wong T., Blitz L., 2002, ApJ, 569, 157

Wright E. L. et al., 2010, AJ, 140, 1868

Wu J., Evans II N. J., Shirley Y. L., Knez C., 2010, ApJS, 188, 313

Wyse R. F. G., 1986, ApJ, 311, L41

Xilouris E. M. et al., 2012, A\&A, 543, A74

York D. G. et al., 2000, AJ, 120, 1579

\section{APPENDIX: FITTING WITH UNIFORM WEIGHTS}

To avoid biasing our data set unnecessarily, we fit to all pixels regardless of S/N. As many of these points (particularly with the molecular gas) are low $\mathrm{S} / \mathrm{N}$, it is important to take into account the errors on these points, and we highlight this here. We have performed a number of simulations where we sample 1000 points distributed normally over the dynamical range of the total gas density, and input a slope $N$ that ranges from 1 to 10 . The points are given scatter in both $\Sigma_{\mathrm{SFR}}$ and $\Sigma_{\text {gas }}$ based on the approximate scatter we see in the data (here, we have assumed normal distributions in both $\Sigma_{\text {SFR }}$ and $\Sigma_{\text {gas }}$ ). We performed a fit in two different ways first, by weighting the errors assuming a constant percentage error on the data, and secondly, by weighting by the RMS errors in our map. For each inputted $N$, we repeat this fit 100 times, and calculate the median recovered $N$, with the 16th and 84th percentiles forming our lower and upper errors. The results of these simulations can be seen in Fig. A1.

For any simulated $N$ above a value of 1 , we see that the uniformly weighted error systematically underestimates the slope. When weighting based on the RMS errors of the map, we can

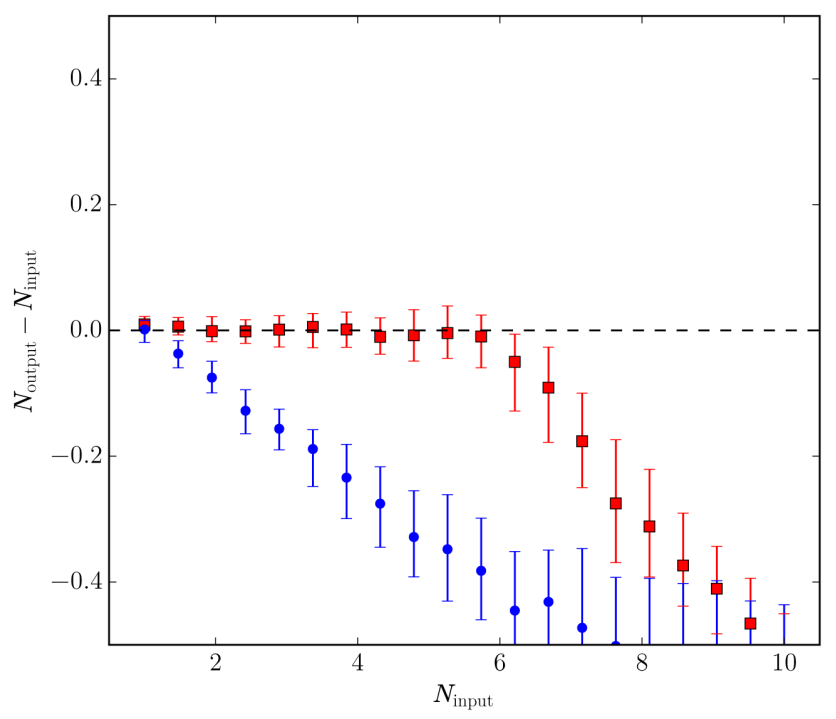

Figure A1. Recovered $N$ against input $N$ for a variety of inputted slopes. The blue dots indicate points where we have assumed a constant percentage error in SFR and gas surface density, the red squares show the errors weighted based on the errors in the relevant maps. The dashed line shows where the recovered slope is equal to the input slope. 
reliably recover slopes up to an $N$ of 6 . This is due to the percentage errors being larger at lower $\mathrm{S} / \mathrm{N}$, and this variation at these low values dominating the fit, especially as the gradient of the slope increases. Since the distribution of $\Sigma_{\text {gas }}$ in the real data is skewed to lower $\mathrm{S} / \mathrm{N}$ rather than normally distributed, we would expect this effect to be even more pronounced in the real data. Realistically accounting for the errors in $\Sigma_{\mathrm{SFR}}$ and $\Sigma_{\text {gas }}$ allows us to recover the underlying Schmidt index of the data across the entire range of $N$ that we calculate in our work, we opt to use this method, rather than assuming a uniform weighting in either $\Sigma_{\mathrm{SFR}}$ or $\Sigma_{\text {gas }}$.

This paper has been typeset from a $\mathrm{TE}_{\mathrm{E}} / \mathrm{LT} \mathrm{E} \mathrm{X}$ file prepared by the author. 